\title{
Collisionality scaling of the electron heat flux in ETG turbulence
}

\author{
G J Colyer ${ }^{1,23}$, A A Schekochihin ${ }^{1,4}$, F I Parra ${ }^{1,2}$, C M Roach ${ }^{2}$, \\ M A Barnes ${ }^{1,2,5}$, Y-c Ghim ${ }^{1,2,6}$ and W Dorland ${ }^{7,1}$ \\ ${ }^{1}$ Rudolf Peierls Centre for Theoretical Physics, University of Oxford, OX1 3NP, UK \\ ${ }^{2}$ CCFE, Culham Science Centre, Abingdon, OX14 3DB, UK \\ ${ }^{3}$ Engineering, Mathematics and Physical Sciences, University of Exeter, EX4 4QF, UK \\ ${ }^{4}$ Merton College, Oxford, OX1 4JD, UK \\ ${ }^{5}$ Plasma Science and Fusion Center, 167 Albany Street, Cambridge, MA 02139, USA \\ ${ }^{6}$ Department of Nuclear and Quantum Engineering, KAIST, Daejeon, 34141, Korea \\ 7 Department of Physics, University of Maryland, College Park, MD 20742-4111, USA
}

\begin{abstract}
In electrostatic simulations of MAST plasma at electron-gyroradius scales, using the local flux-tube gyrokinetic code GS2 with adiabatic ions, we find that the longtime saturated electron heat flux (the level most relevant to energy transport) decreases as the electron collisionality decreases. At early simulation times, the heat flux "quasisaturates" without any strong dependence on collisionality, and with the turbulence dominated by streamer-like radially elongated structures. However, the zonal fluctuation component continues to grow slowly until much later times, eventually leading to a new saturated state dominated by zonal modes and with the heat flux proportional to the collision rate, in approximate agreement with the experimentally observed collisionality scaling of the energy confinement in MAST. We outline an explanation of this effect based on a model of ETG turbulence dominated by zonal-nonzonal interactions and on an analytically derived scaling of the zonal-mode damping rate with the electron-ion collisionality. Improved energy confinement with decreasing collisionality is favourable towards the performance of future, hotter devices.
\end{abstract}

\section{Introduction}

Experiments on MAST in which heat transport is dominated by the electron channel find that the thermal energy confinement time $\tau_{E}$ varies with the normalised electron collisionality $\nu_{*}$ according to the scaling [1]

$$
B \tau_{E} \propto \nu_{*}^{-0.82 \pm 0.1}
$$

where $B$ is the magnetic field. This scaling is favourable towards improved confinement in the hotter, lower collisionality plasmas anticipated in future devices.

In this paper, we investigate how the electron heat flux $Q$ varies with electron collisionality in simulations of electron-scale plasma turbulence in MAST, using the local gyrokinetic flux-tube 
code GS2 $[2,3]$. At constant geometry and $\rho_{*}=\rho_{e} / a\left(\rho_{e}\right.$ is the electron Larmor radius, $a$ is the equilibrium length scale), it can be shown (see Appendix A) that

$$
B \tau_{E} \propto\left(\frac{Q}{Q_{\mathrm{gB}}}\right)^{-1},
$$

where $Q_{\mathrm{gB}}=n T v_{t e} \rho_{*}^{2}$ is the electron gyroBohm heat flux. It therefore ought to be possible to recover the scaling (1) from a local calculation of the electron heat flux.

We wish to discover whether this experimental MAST scaling may be understood in terms of electron temperature gradient (ETG) driven turbulent transport. With this goal in mind, we carry out local gyrokinetic simulations restricted to electrostatic perturbations at electron-gyroradius scales, ignoring both electromagnetic (e.g., "microtearing" [4, 5]) modes and ion-gyroradius-scale effects. The restriction to electrostatic perturbations is a matter of considering as simple a model as possible for a plasma where beta is low and magnetic perturbations are relatively small. The neglect of the ion-gyroradius-scale turbulence is justifiable on the grounds that, in typical MAST plasmas, ion turbulence is considerably suppressed by radial shear in the background flow, and ion transport is close to the neoclassical level $[6,7,8,9]$. This is fortunate, as spanning both ionand electron-gyroradius scales requires prohibitively large computational resources. We further limit ourselves here to a Boltzmann-ion model, at electron scales, treating only the electrons kinetically. Whilst greatly simplified, the equations we solve (which are described in more detail in section 2) reproduce the experimental scaling of electron heat flux with collisionality.

The reference simulation parameters are based on experiment, which is close to the threshold for the onset of turbulence. In this region of parameter space, we find that the saturated turbulent heat flux varies with collisionality in a manner consistent with the experimental scaling. This scaling is only revealed, however, if the simulation times are sufficiently long to reach a true steady state, which requires them to be much longer than the electron-collision time scale. At earlier times, there is a transient "quasi-saturated" state with higher heat flux, in which the zonal

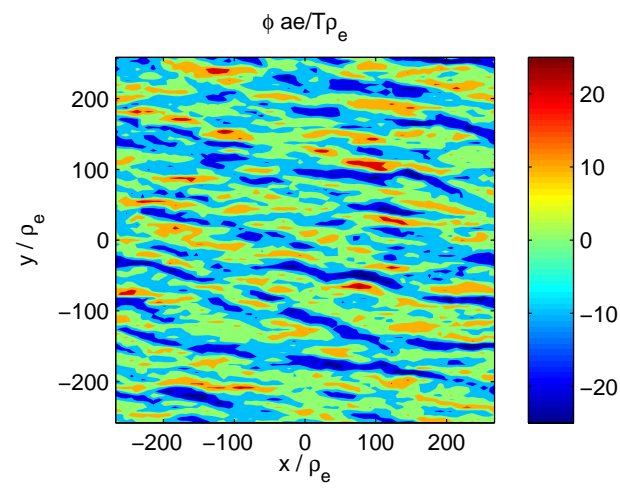

(a)

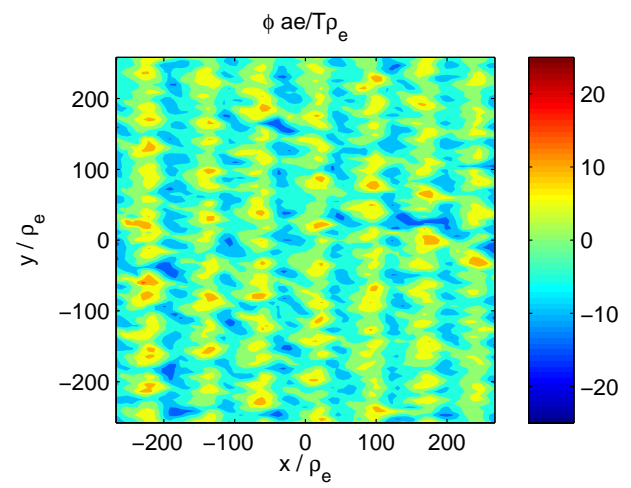

(b)

Figure 1. Non-dimensionalised electrostatic potential $e \phi / T \rho_{*}$ (where $\rho_{*}=\rho_{e} / a$ ) at the outboard midplane, for $\nu=0.2 \nu_{\text {nom }}$ (here $\nu_{\text {nom }}$ is the "nominal", i.e., experimental value of collisionality), $a / L_{T}=3.3$ : (a) quasi-saturated state at $t=1200.3 a / v_{t e}$, (b) saturated state at $t=7835.8 a / v_{t e}$, for large-box simulations. See Appendix B and Appendix C for the meaning of symbols. 
modes (which do not themselves contribute to the heat flux) are small but slowly growing. When they have grown to a sufficient level, the nonzonal modes and the heat flux are significantly suppressed. Figure 1 illustrates these two regimes by showing the electrostatic potential (which is proportional to the density perturbation) in an outboard-midplane cross-section of a flux tube in MAST both in the earlier quasi-saturated state and the later long-time saturated state, based on one of the simulations reported below. In the quasi-saturated state, the zonal modes do not appear to play a special role, and radially extended "streamers" can be seen, as is usually expected for ETG turbulence [10, 11]. In contrast, in the long-time saturated state, a strong zonal component comes to dominate, structuring the turbulence into "vortex streets" and dramatically weakening radial transport. In this final saturated state, the nonlinear drive of the zonal modes is balanced by their weak collisional damping, dominated by electron-ion collisions. Scans in collisionality reveal that the saturated heat flux increases with increasing collisionality, in rough proportionality: $Q / Q_{\mathrm{gB}} \propto \nu_{*}$.

A brief outline of the rest of the paper is as follows. In section 2, we describe the equations that are solved and the simulation set-up. In section 3, we present our main results, including the long-time evolution of the turbulence, the dependence of the saturated heat flux on collisionality, and the structure of the saturated turbulent state. We also sketch a simple theoretical argument that explains the collisionality scaling of the heat flux (section 3.2). In section 4, a summary of our findings is given, our results are put in the context of some earlier work, and a discussion is given of the apparent differences and possible similarities between the ETG and ITG turbulent states in light of the conclusions of the present study.

\section{Governing equations and numerical set-up}

Our study is based on the MAST H-mode shot 8500, which had 2 MW of NBI heating, and for which data are available from the International Tokamak Profile Database [12]. This shot was analysed by Field et al. [6], and a linear gyrokinetic study was performed by Roach et al. [7]. In the present work, we consider a single flux surface, for which the detailed plasma parameters are given in Appendix B (they are referred to as "nominal" parameters); these were kept fixed throughout our study, except for varying collisionality and electron temperature gradient where indicated. In Appendix C, we provide the technical details about our numerical simulations: the coordinate system used, numerical grids, resolution and boundary conditions.

\subsection{Gyrokinetic equation}

We use the GS2 continuum gyrokinetic code [3] to obtain the perturbed distribution function and electrostatic field in local flux-tube geometry. The electron distribution function is written

$$
f=F+\delta f
$$

(we omit species subscripts when this will cause no confusion), where

$$
F=n\left(\frac{m}{2 \pi T}\right)^{3 / 2} \exp \left(-\frac{m v^{2}}{2 T}\right)
$$

is the equilibrium Maxwellian background distribution, $n, m, T$ and $\mathbf{v}$ are the electron density, mass, temperature and velocity, respectively, and the perturbed distribution function is split into 
a Boltzmann response associated with the perturbed electrostatic potential $\phi$ and a gyrotropic non-Boltzmann, generally non-Maxwellian part:

$$
\delta f=\frac{e \phi(\mathbf{r})}{T} F+h\left(\mathbf{R}, v_{\perp}, v_{\|}\right)
$$

where $e$ is the absolute value of the electron charge. Note that $\phi$ is a function of the particle position $\mathbf{r}$, whereas $h$ is a function of the gyrocentre position $\mathbf{R}=\mathbf{r}-\mathbf{b} \times \mathbf{v} / \Omega_{e}$, where $\mathbf{b}$ is the unit vector along the magnetic field and $\Omega_{e}=-e B / m c$ is the electron cyclotron frequency (its sign is the negative sign of the electron charge). The gyrocentre distribution $h$ is otherwise independent of the gyroangle, being a function of the parallel $v_{\|}=\mathbf{v} \cdot \mathbf{b}$ and perpendicular $v_{\perp}=\left(v^{2}-v_{\|}^{2}\right)^{1 / 2}$ velocities. Equivalently, its velocity-space variables can be (and are) chosen to be the energy $\mathcal{E}=v^{2} / 2$ and magnetic moment $\mu=v_{\perp}^{2} / 2 B$, so $v_{\|}= \pm(2 \mathcal{E}-2 \mu B)^{1 / 2}$. In this representation, the gyrocentre distribution $h$ satisfies the electrostatic gyrokinetic equation (GKE) [13] (see review [14])

$$
\frac{\partial}{\partial t}\left(h+\frac{e\langle\phi\rangle}{T} F\right)+\left(v_{\|} \mathbf{b}+\mathbf{v}_{B}\right) \cdot \nabla h+\left\langle\mathbf{v}_{E}\right\rangle \cdot \nabla h+\left\langle\mathbf{v}_{E}\right\rangle \cdot \nabla F=\langle C[h]\rangle,
$$

where $\langle\ldots\rangle$ denotes gyroaveraging at constant gyrocentre position $\mathbf{R}$,

$$
\mathbf{v}_{B}=\frac{\mathbf{b}}{\Omega_{e}} \times\left(v_{\|}^{2} \mathbf{b} \cdot \nabla \mathbf{b}+\frac{v_{\perp}^{2}}{2} \frac{\nabla B}{B}\right)
$$

is the magnetic drift velocity, and

$$
\mathbf{v}_{E}=\frac{c}{B} \mathbf{b} \times \nabla \phi
$$

is the $\mathbf{E} \times \mathbf{B}$ drift velocity. The energy is injected into the system via the last term on the left-hand side of (6), which contains the radial gradients of the equilibrium distribution:

$$
\left\langle\mathbf{v}_{E}\right\rangle \cdot \nabla F=\left\langle v_{E x}\right\rangle \frac{\partial F}{\partial x}=\left\langle v_{E x}\right\rangle\left[\frac{1}{L_{n}}+\left(\frac{v^{2}}{v_{t e}^{2}}-\frac{3}{2}\right) \frac{1}{L_{T}}\right] F,
$$

where $v_{t e}=(2 T / m)^{1 / 2}$ is the electron thermal speed. We have defined $L_{n}=-d \ln n / d x$ and $L_{T}=-d \ln T / d x$, the gradient scale lengths of the equlibrium electron density and temperature profiles, respectively. The natural normalisation of these lengths is the tokamak minor radius a. In the above definitions, $x$ is the spatial coordinate transverse to the flux surface and $y$ is a coordinate within the flux surface transverse to the magnetic field (at the outboard midplane, it is approximately the poloidal arc length) — these curvilinear, non-orthogonal coordinates, as used by GS2, are explained in Appendix C.1. Note that, in these coordinates,

$$
v_{E x}=\mathbf{v}_{E} \cdot \nabla x=\frac{c q}{B_{0}^{2} r} \frac{d \psi}{d r} \frac{\partial \phi}{\partial y}
$$

(see Appendix C.1 for the definition of all symbols).

\subsection{Adiabatic ions}

In a simple (two-species, hydrogenic, i.e., with ion charge $=+e$ ) plasma, the quasineutrality condition for the perturbations is

$$
\delta n_{e}=\delta n_{i},
$$


where $\delta n=\int d^{3} \mathbf{v} \delta f$ is the density perturbation for each species. In terms of $h$, this density perturbation is

$$
\frac{\delta n}{n}=-\frac{Z e \phi}{T}+\frac{1}{n} \int d^{3} \mathbf{v}\langle h\rangle_{\mathbf{r}}
$$

where $\langle\cdot\rangle_{\mathbf{r}}$ denotes the gyroaveraging operator at constant particle position $\mathbf{r}$ (needed here because the velocity integral must be performed at fixed particle position $\mathbf{r}$, while $h$ is a function of $\mathbf{R}$ ), and $Z=1$ for the ions and -1 for the electrons. We have assumed a decomposition of the perturbed ion distribution function analogous to (5). If we consider only perturbations at scales perpendicular to the magnetic field that are much smaller than the ion gyroradius, $k_{\perp} \rho_{i} \gg 1$, the non-Boltzmann part of the ion density response in (12) can be neglected because $\left\langle h_{i}\right\rangle_{\mathbf{r}}$ is suppressed by the averaging over large ion Larmor orbits. In formal terms, this approximation is the lowest-order expansion in the electron-ion mass ratio. Therefore, in the present study, we will assume that the ion distribution is entirely described by the Boltzmann (sometimes called adiabatic) response:

$$
\frac{\delta n_{i}}{n_{i}}=-\frac{e \phi}{T_{i}} .
$$

Combining this with the quasineutrality (11) and the full gyrokinetic electron density response given by (12) with $Z=-1$, we get the following equation for $\phi$ :

$$
\frac{e \phi}{T_{e}}\left(1+\frac{1}{\tau}\right)=-\frac{1}{n} \int d^{3} \mathbf{v}\langle h\rangle_{\mathbf{r}}
$$

where $h$ satisfies (6) and $\tau=T_{i} / T_{e}$.

Equations (6) and (14) constitute a closed system, which is solved numerically by the version of the GS2 code that we use here.

\subsection{Collisions}

The electron collision operator used in GS2, appearing on the right-hand side of (6), consists of the electron-ion pitch-angle scattering operator (to lowest order in the mass-ratio expansion) and a particle-, momentum- and energy-conserving simplified model of the electron-electron collision operator $[7,15,16]$ : in $\mathbf{k}_{\perp}$ space, it is

$$
\langle C[h]\rangle_{\mathbf{k}_{\perp}}=\left\langle C_{e e}[h]\right\rangle_{\mathbf{k}_{\perp}}+\nu_{e i} \frac{v_{t e}^{3}}{v^{3}}\left[\frac{\partial}{\partial \xi} \frac{\left(1-\xi^{2}\right)}{2} \frac{\partial h_{\mathbf{k}_{\perp}}}{\partial \xi}-\frac{\left(1+\xi^{2}\right)}{4} \frac{v^{2}}{v_{t e}^{2}} k_{\perp}^{2} \rho_{e}^{2} h_{\mathbf{k}_{\perp}}\right],
$$

where $\xi=v_{\|} / v$, the electron-electron operator $\left\langle C_{e e}[h]\right\rangle$ is given in [15] and the numerical implementation of the whole operator is detailed in [16]. $\ddagger$ The electron-ion collision rate is $\nu_{e i}=Z_{\text {eff }} \nu_{e e}$, where $\nu_{e e} \equiv \nu=\sqrt{2} \pi n e^{4} T^{-3 / 2} m^{-1 / 2} \ln \Lambda$ is the electron-electron collision rate and $\ln \Lambda$ is the Coulomb logarithm. In a plasma with multiple ion species, $Z_{\mathrm{eff}}=\sum_{i} n_{i} Z_{i}^{2} / \sum_{i} n_{i} Z_{i}$ arises from summing the individual electron-ion collision operators over all ion species $\left(Z_{i} e\right.$ is that species' charge); in a hydrogenic plasma, $Z_{\mathrm{eff}}=1$. Note, however, that GS2 treats $Z_{\mathrm{eff}}$ as an

‡ As originally written, GS2 was not configured to simulate kinetic electrons with adiabatic (Boltzmann) ions and also to include electron-ion collisions. This is because GS2 always formally requires at least one kinetic ion species, and so single-species KE+AI simulations were actually performed as single-species kinetic-ions simulations with the sign of the charge in the Boltzmann response appropriately reversed. The equations solved are then correct for simulations with kinetic electrons and adiabatic ions without collisions or with same-species collisions only. For the present work, we added the capability to include the electron-ion collision term in such simulations; previously it was included only for electrons in multi-species simulations with both kinetic ions and kinetic electrons. 
independent parameter in equation (15), formally allowing one to vary $\nu_{e i}$ relative to $\nu_{e e}$ without affecting the quasineutrality equation (11). This is normally done to include the effect of electron collisions with impurity ion species that are not being modelled kinetically, but are present in real experiments. In this work, it will also be useful to us in section 3.5 as a method of varying this ratio artificially.

Electron-ion collisions relax the parallel electron flow towards the stationary ion background, resistively dissipating the current associated with an electron flow below the ion gyroscale. $\S$ As a result, the electron-ion collision operator, and hence the electron collision operator overall, does not conserve (electron) momentum. || The last term in the bracket in (15) is a spatial diffusion it is a finite-Larmor-radius (FLR) effect arising from the gyroaveraging of the collision operator $[15]$.

\section{Results}

\subsection{Time evolution: from "quasi-saturation" to long-time steady state}

Figure 2(a) shows the turbulent electron heat flux as a function of time for two simulations that have identical plasma parameters and differ only in numerical grid parameters (their coincidence is evidence of numerical convergence; for more details of the numerics and of the various issues of convergence, see Appendix E, which also contains plots for other values of $\nu$ and $a / L_{T}$ and of other zonal quantities). The heat flux $Q$ is calculated from the solution of equations (6) and (14) according to

$$
Q=\overline{\int d^{3} \mathbf{v} \frac{m v^{2}}{2} \delta f v_{E x}},
$$

where the overbar indicates a flux-surface average. We will normalise $Q$ to the gyroBohm value $Q_{\mathrm{gB}}=n T v_{t e} \rho_{*}^{2}$, where $\rho_{*}=\rho_{e} / a$. Besides its physical meaning as the heat flux, $Q$ is also a good proxy for the turbulent fluctuation level of the non-zonal modes $\left(k_{y} \neq 0\right.$; the $k_{y}=0$ components of $\phi$ or $h$ do not contribute to $Q$ ).

After a short exponential transient during the linear growth phase, the system reaches a "quasisaturated" turbulent state, which, however, is not the final steady state. The final saturated state is reached much later, after a slow decline in heat flux accompanied by slow growth of the zonal $\left(k_{y}=0\right)$ component of the turbulence: Figure 2(b) shows the evolution of the zonal velocity squared,

$$
\left(k_{\mathrm{Z}} \phi_{\mathrm{Z}}\right)^{2}=\sum_{k_{x}} k_{x}^{2}\left|\phi_{k_{x}, 0}\right|^{2}
$$

(this equation also introduces the definition of the characteristic zonal scale $k_{\mathrm{Z}}$ ). Snapshots of $\phi$, shown in Figure 1 and corresponding to early and late times in one of these simulations, are a vivid illustration of the different structure of the "quasi-saturated" and the final steady state: the former resembles the streamer-dominated state usually associated with ETG turbulence [10, 11], whereas the latter is a zonal-mode-dominated state, which we will now proceed to analyse. Note

$\S$ The electron flow is also a current because, in the limit of $k_{\perp} \rho_{i} \gg 1$, ion gyromotion averages out over the electron scales, leaving, to lowest order, no ion-flow response in either parallel or perpendicular direction; cf. the adiabatic-ion approximation (13), which arises from the averaging out of the gyrokinetic ion-density response.

\| As we see in section 3, electron-ion collisions reduce the final saturation amplitude of electron-scale zonal flows, and this gives rise to a favourable scaling of electron heat flux with collisionality. 


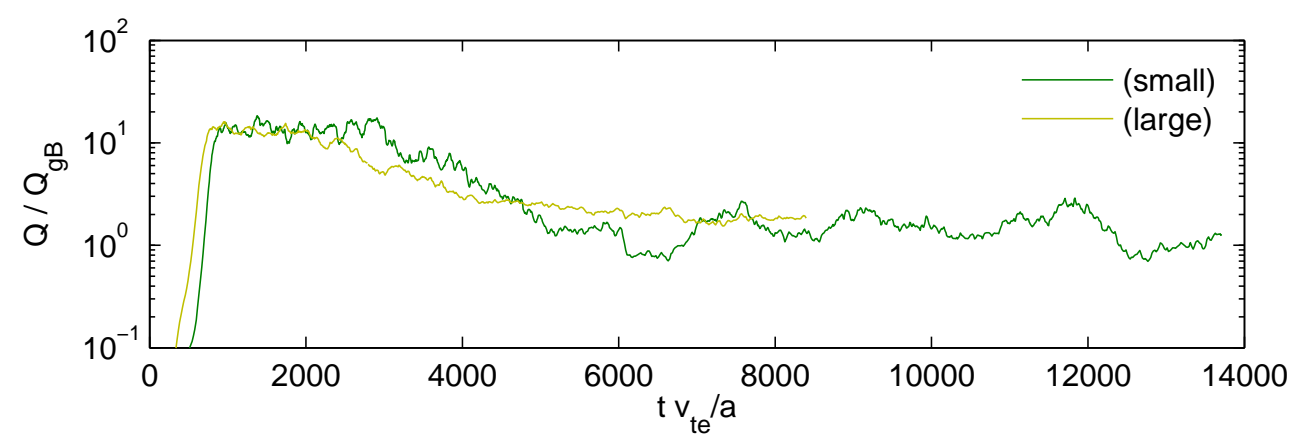

(a)

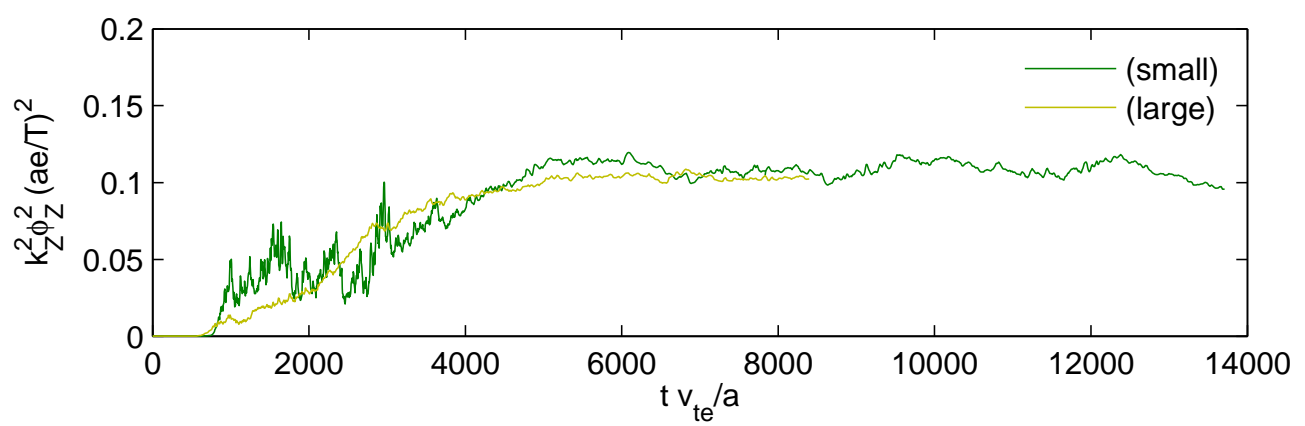

(b)

Figure 2. Evolution in time of (a) the turbulent electron heat flux in electron gyroBohm units, and (b) the square of the zonal velocity, $\left(k_{\mathrm{Z}} \phi_{\mathrm{Z}}\right)^{2}$, for two simulations with electron collisionality $\nu=0.2 \nu_{\text {nom }}$, electron temperature gradient $a / L_{T}=3.3$ (green: small-box simulation; yellow: large-box simulation; see Appendix C.2 for details).

that it is this long-time saturated state that matters for determining the level of transport because, even though the time for it to emerge is long by the standards of electron-gyroscale dynamics, it is still much shorter than the transport time scale in a tokamak. $\uparrow$ This is because the self-consistent combination of $\delta f$ local (gradient-driven) gyrokinetics at the gyroradius scale, and radial transport evolution at the system scale, implies a scale separation in both space and time $[17,14]$.

\subsection{Collisionality scaling: numerical results and theory}

The two clearest results from the final saturated state of these simulations are summarised in Figure 3, which is based on a parameter scan in electron collisionality for two values of the electron temperature gradient, at two different numerical resolutions. The saturated heat flux increases roughly proportionally to the collisionality, whereas the zonal velocity is essentially independent of it. The heat-flux scaling is in remarkable agreement with the experimental scaling (1). ${ }^{+}$

I Using MAST data from [1], $\tau_{E}$ is of order $10 \mathrm{~ms}$, whereas $a / v_{t e}$ is of order $10 \mathrm{~ns}$, a factor of $10^{6}$ smaller. The longest runs in this paper evolve for times only of order $10^{4} a / v_{t e}$, so there is still good scale separation.

+ To facilitate a quantitative comparison of our results with [1] - whilst noting that we consider a particular flux surface whereas the authors of [1] considered the plasma globally - we convert our nominal $\nu=0.02 v_{t e} / a$ (Table B1) using the expression (A.5) to get a nominal $\nu_{*} \approx 0.1$. The collisionality range shown in our Figure 3 is thus 


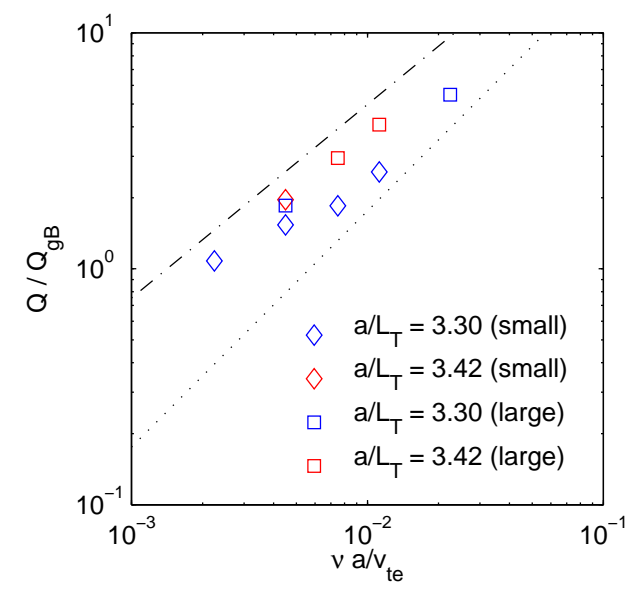

(a)

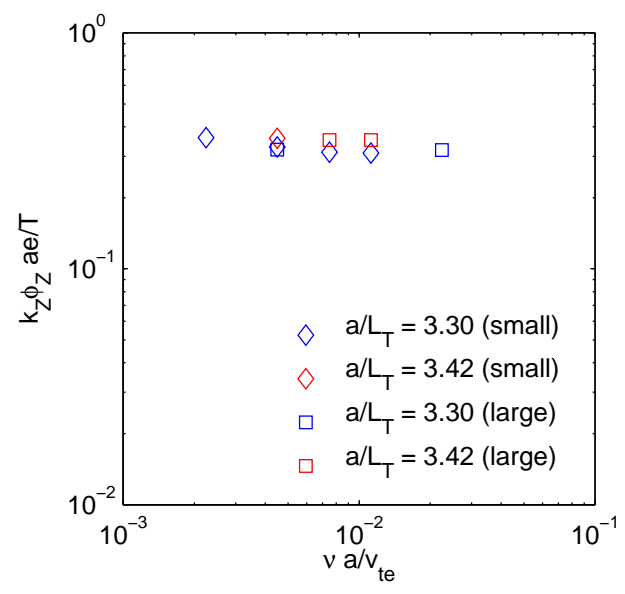

(b)

Figure 3. Variation of (a) the time-averaged normalised electron heat flux $Q / Q_{\mathrm{gB}}$, and (b) the rms zonal velocity $k_{\mathrm{Z}} \phi_{\mathrm{Z}}$, defined by (17), versus normalised electron collisionality $\nu a / v_{t e}$, at the nominal (experimental) value of the temperature gradient $a / L_{T}=3.42$, and at $a / L_{T}=3.3$. Symbol shapes indicate the simulation series ("small box" or "large box"), as explained in Appendix E. The dot-dashed line shows the experimental power-law scaling, and the dotted line shows the theoretical linear scaling, $Q / Q_{\mathrm{gB}} \propto \nu_{*}$, equation (31).

Let us outline a simple explanation of these results, which we will then follow up with a series of numerical experiments designed to test its plausibility.

Let us split the gyrokinetic equation (6) explicitly into equations governing the evolution of the nonzonal and zonal components of the distribution function and the associated electrostatic potential (cf. [18]),

$$
h=h_{\mathrm{NZ}}+h_{\mathrm{Z}}, \quad \phi=\phi_{\mathrm{NZ}}+\phi_{\mathrm{Z}},
$$

where the subscripts NZ, Z denote nonzonal $\left(k_{y} \neq 0\right)$ and zonal $\left(k_{y}=0\right)$ modes, respectively:

$$
\begin{aligned}
& \frac{\partial}{\partial t}(\left.h_{\mathrm{NZ}}+\frac{e\left\langle\phi_{\mathrm{NZ}}\right\rangle}{T} F\right)+\left(v_{\|} \mathbf{b}+\mathbf{v}_{B}\right) \cdot \nabla h_{\mathrm{NZ}}-\left\langle C\left[h_{\mathrm{NZ}}\right]\right\rangle \\
&+\underbrace{\left\langle\mathbf{v}_{E, \mathrm{NZ}}\right\rangle \cdot \nabla h_{\mathrm{Z}}+\left\langle\mathbf{v}_{E, \mathrm{Z}}\right\rangle \cdot \nabla h_{\mathrm{NZ}}}_{\mathrm{Z}-\mathrm{NZ} \text { interaction (I) }}+\left\langle\mathbf{v}_{E, \mathrm{NZ}}\right\rangle \cdot \nabla h_{\mathrm{NZ}}-\overline{\left\langle\mathbf{v}_{E, \mathrm{NZ}}\right\rangle \cdot \nabla h_{\mathrm{NZ}}} \\
&=\underbrace{-\left\langle\mathbf{v}_{E, \mathrm{NZ}}\right\rangle \cdot \nabla F}_{\text {energy injection (II) }}, \\
& \frac{\partial}{\partial t}\left(h_{\mathrm{Z}}+\frac{e\left\langle\phi_{\mathrm{Z}}\right\rangle}{T} F\right)+\left(v_{\|} \mathbf{b}+\mathbf{v}_{B}\right) \cdot \nabla h_{\mathrm{Z}}-\underbrace{\left\langle C\left[h_{\mathrm{Z}}\right]\right\rangle}_{\text {damping (III) }}=\underbrace{-\overline{\left\langle\mathbf{v}_{E, \mathrm{NZ}}\right\rangle \cdot \nabla h_{\mathrm{NZ}}}}_{\text {energy injection (IV) }},
\end{aligned}
$$

where the overline denotes spatial averaging over $y$, i.e., the $k_{y}=0$ component. Equation (20) is the $y$ average of the gyrokinetic equation (6); then (19) is the result of subtracting (20) from $(6)$.

approximately the same as the range of $\nu_{*}$ accessed experimentally (approximately $0.03-0.1$ in Figure 3 of [1]) for which the scaling (1) was obtained. 
We conjecture that the dominant balance governing the saturated state of the nonzonal modes is between the zonal-nonzonal interaction terms (I) and the the linear drive (energy-injection) term (II) in (19). $\dagger$ We estimate these terms as

$$
(\mathrm{I}) \sim\left\langle\mathbf{v}_{E, \mathrm{NZ}}\right\rangle \cdot \nabla h_{\mathrm{Z}} \sim \frac{c}{B} k_{y} \phi_{\mathrm{NZ}} k_{\mathrm{Z}} h_{\mathrm{Z}}
$$

and

$$
(\mathrm{II}) \sim\left\langle\mathbf{v}_{E, \mathrm{NZ}}\right\rangle \cdot \nabla F \sim \frac{c}{B} k_{y} \phi_{\mathrm{NZ}} \frac{F}{L_{T}}
$$

where $k_{\mathrm{Z}}$ is the typical zonal wavenumber and $k_{y}$ the typical nonzonal wavenumber (we have used $v_{E x} \sim(c / B) k_{y} \phi$; see (10)). The second zonal-nonzonal interaction term, $\left\langle\mathbf{v}_{E, \mathrm{Z}}\right\rangle \cdot \nabla h_{\mathrm{NZ}}$, is of the same order as (21) if we assume that

$$
\frac{h}{F} \sim \frac{e \phi}{T}
$$

for both zonal and nonzonal modes. Balancing (21) and (22), we find, after cancellation of $k_{y} \phi_{\mathrm{NZ}}$, that

$$
k_{\mathrm{Z}} h_{\mathrm{Z}} \sim \frac{F}{L_{T}} .
$$

This is a form of mixing-length hypothesis [22], suggesting that the perturbed zonal gradients $\sim k_{\mathrm{Z}} h_{\mathrm{Z}}$ compensate the background equilibrium gradients associated with $F . \ddagger$ It follows from (23) and (24) that

$$
k_{\mathrm{Z}} \frac{e \phi_{\mathrm{Z}}}{T} \sim \frac{1}{L_{T}}
$$

Thus, the gradients of the zonal modes (e.g., the zonal velocity or the zonal temperature gradient) are independent of collisionality. This independence is indeed seen in Figure 3(b).

The only nonlinearity present in the zonal equation (20) is the nonzonal-nonzonal interaction term (IV); zonal modes are not directly driven by background gradients because $\nabla F$ is in the $x$ direction. One can think of (20) as a kind of Langevin equation for zonal modes, which are excited by coupling between nonzonal modes and damped by collisions (at long times, the only damping mechanism is collisional; see Appendix F).§ Therefore, the dominant balance in (20) is between the nonlinear energy injection (IV) and collisional damping (III):

$$
\frac{c}{B} k_{\mathrm{Z}} k_{y} \phi_{\mathrm{NZ}} h_{\mathrm{NZ}} \sim \gamma_{\mathrm{Z}} h_{\mathrm{Z}}
$$

$\dagger$ We are thus treating the collision term in the nonzonal equation as subdominant, or at least as unimportant to this aspect of the dynamics. Numerically we find that it cannot be neglected as it regularises the fine velocity-space structure arising due to the phase-mixing of $h_{\mathrm{NZ}}$ [19]. The parallel streaming and magnetic-drift terms likely play a part in determining the spatial structure of the turbulence $[20,21,19]$, but we shall see that we do not need to determine $k_{y}, k_{\mathrm{Z}}$ or $k_{\|}$. In [18], a split between nonzonal and zonal components is performed for integrated entropy balance equations (equations (67) and (68) of [18]) in which these other terms do not appear. One could base a similar argument to the one presented here on these equations.

‡ They need not necessarily flatten the background gradient completely or everywhere. From Figure 2(b), the normalised zonal velocity $k_{\mathrm{Z}} \rho_{e} e \phi_{\mathrm{Z}} / T \rho_{*} \approx 0.3$ (we also find $k_{\mathrm{Z}} \rho_{e} \delta T_{\mathrm{Z}} / T \rho_{*} \approx 0.3$ ), which should be compared with the background gradient $a / L_{T}=3.3$. For the (random-noise) initial conditions used in our simulations, the minimum $a / L_{T}$ required to sustain turbulence is between 3.0 and 3.3, so the perturbation levels correspond roughly to the distance away from this nonlinear critical gradient (of which we have not made a precise determination because the system appears to be strictly subcritical in the presence of even small flow shear; the linear critical gradient without flow shear is about 2.4, as shown in Appendix D). Furthermore, the perturbed gradient is not constant ( $i n c e k_{\mathrm{Z}} \neq 0$ ); the actual magnitude of the gradient at any particular $x$ can exceed 0.3 .

$\S$ How precisely the zonal modes are generated is a matter for future research, but see section 3.6. 
where $\gamma_{Z}$ is the collisional damping rate of the zonal modes. Note that in conjecturing such a balance, we are assuming the zonal modes are not subject to a strong nonlinear instability that would break them down back into nonzonal perturbations, thus resulting in a purely collisionless saturated state. This possibility (which, for example, appears to be realised for zonal flows in certain regimes of ITG turbulence, where it is known as the tertiary instability [23]) would amount to a dominant balance in (20) between the energy-injection and energy-removal effects within the nonlinear term (IV). We are expressly assuming that this is not the dominant balance in our near-marginal ETG turbulence.

Combining equations (25), (26), and (23) again, we get

$$
\frac{h_{\mathrm{NZ}}^{2}}{h_{\mathrm{Z}}^{2}} \sim \frac{\phi_{\mathrm{NZ}}^{2}}{\phi_{\mathrm{Z}}^{2}} \sim \frac{\gamma_{\mathrm{Z}} e B L_{T}}{c k_{y} T} \sim \frac{1}{k_{y} \rho_{e}} \frac{\gamma_{\mathrm{Z}}}{v_{t e} / L_{T}} .
$$

Therefore, estimating the heat flux (16), we find

$$
\frac{Q}{Q_{\mathrm{gB}}} \sim \frac{n \delta T_{\mathrm{NZ}} v_{E x}}{n T v_{t e} \rho_{*}^{2}} \sim \frac{\delta T_{\mathrm{NZ}}}{T} \frac{c k_{y} \phi_{\mathrm{NZ}}}{B v_{t e} \rho_{*}^{2}} \sim k_{y} \rho_{e}\left(\frac{e \phi_{\mathrm{NZ}}}{T \rho_{*}}\right)^{2},
$$

where we have used (23) to estimate $\delta T / T \sim e \phi / T$. Using (27) to relate $\phi_{\mathrm{NZ}}^{2}$ to $\phi_{\mathrm{Z}}^{2}$ and $(25)$ to estimate $\phi_{\mathrm{Z}}^{2}$, we get

$$
\frac{Q}{Q_{\mathrm{gB}}} \sim \frac{\gamma_{\mathrm{Z}}}{v_{t e} / L_{T}}\left(\frac{e \phi_{\mathrm{Z}}}{T \rho_{*}}\right)^{2} \sim \frac{\gamma_{\mathrm{Z}}}{k_{\mathrm{Z}}^{2} \rho_{e}^{2}\left(v_{t e} / a\right)} \frac{a}{L_{T}} .
$$

Thus, we are able to estimate the electron heat conductivity entirely in terms of the linear damping rate and characteristic scale of the zonal flows. It is possible to show analytically (Appendix F) and confirm numerically (section 3.3) that in the long term, zonal modes are damped by collisional (Ohmic) resistivity:

$$
\gamma_{\mathrm{Z}} \sim \nu_{e i} k_{\mathrm{Z}}^{2} \rho_{p e}^{2}
$$

where $\nu_{e i}$ is the electron-ion collision rate and $\rho_{p e}=\rho_{e} B / B_{p}$ is the "poloidal Larmor radius" of the electrons $\left(B_{p}\right.$ is the poloidal magnetic field; see Appendix F.4 for a more precise definition of $\left.\rho_{p e}\right)$. Using (30) in (29), we finally obtain

$$
\frac{Q}{Q_{\mathrm{gB}}} \sim \frac{\nu_{e i}}{v_{t e} / a}\left(\frac{B}{B_{p}}\right)^{2} \frac{a}{L_{T}} \propto \nu_{e i}
$$

Thus, a relatively simple theoretical argument has produced a linear scaling of the heat flux with collisionality. Note that all dependence on $k_{\mathrm{Z}}$ or any other wave numbers has cancelled in the final expression (31), and so, in order to obtain the heat flux, we need not know the spatial scales of either zonal or nonzonal modes. Considering the simplicity of the argument, and the level of agreement between it, our numerical results (Figure 3), and the experimental MAST scaling (1), we find it quite compelling.

\subsection{Damping of zonal modes}

In the theoretical argument of section 3.2, a crucial step was to use the expression (30) for the collisional damping of the zonal modes, which allowed us to estimate the heat flux according to (29) and avoid having to theorise about the characteristic scale of the zonal modes (a nontrivial question, with, as our simulations indicate, possibly a nonuniversal answer). In Appendix F, 


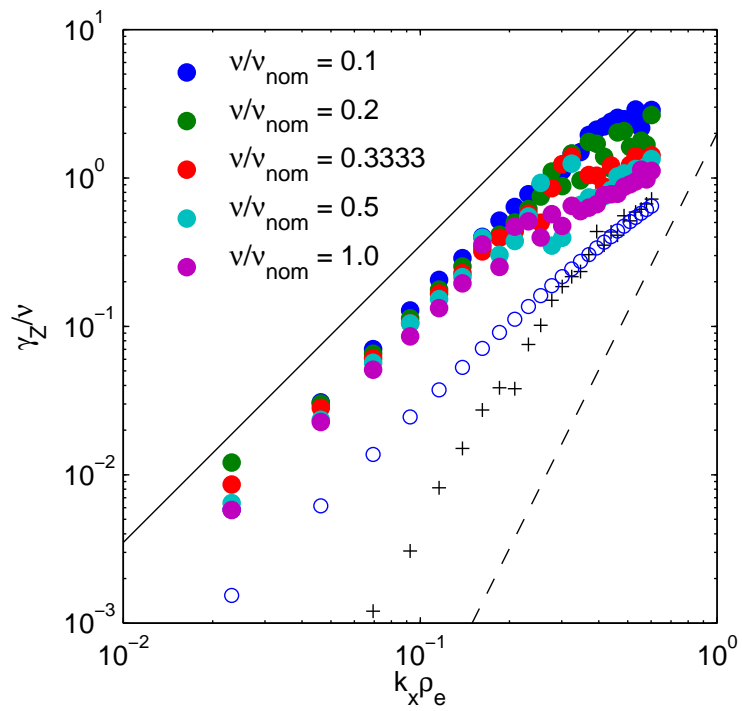

Figure 4. Zonal damping rate normalised to collisionality, $\gamma_{\mathrm{Z}} / \nu$, versus $k_{x} \rho_{e}$, spanning the range of collisionalities shown in Figure 3 (solid colors). The final states of various saturated nonlinear simulations were used as initial conditions, with the nonlinearity switched off. Also shown (black crosses) are the corresponding damping rates for a simulation at $\nu=\nu_{\text {nom }}$ in which electron-ion collisions were turned off (formally by setting $Z_{\text {eff }}=0$; see section 2.3 ); and a simulation at $\nu=\nu_{\text {nom }}$ in which electron-ion collisions were retained but magnetic drifts were turned off (blue open circles). The solid line is the slope $\propto k_{x}^{2}$, corresponding to the scaling (30); the dashed line is $\propto k_{x}^{4}$, corresponding to the scaling expected when $\nu_{e i}=0$ (see Appendix F.7).

the damping rate (30) is derived analytically. Physically, the situation can be summarised as follows.

Consider a zonal perturbation with some perpendicular wave number $k_{x}$ satisfying (20) with zero right-hand side - a linear equation. In the absence of collisions, this perturbation will decay quickly (on the time scale $\sim a / v_{t e}$ ), but not to zero, leaving a finite residual zonal field [24]. With collisions present, after a period of a few collision times, which is still much shorter than the damping time, $\nu^{-1} \ll \gamma_{\mathrm{Z}}^{-1} \sim\left(\nu k_{x}^{2} \rho_{e}^{2}\right)^{-1}$ in the long-wavelength limit $k_{x} \rho_{e} \ll 1$, it is possible to show that, to lowest order in $k_{x} \rho_{e}$, the remaining perturbation is a perturbed Maxwellian with a density (or, equivalently, $\phi$ ) and a temperature perturbation, both constant on each flux surface. These perturbations then decay diffusively due to perpendicular particle diffusion (equivalently, resistivity) arising from the collision operator. We already saw in section 2.3 that the gyrokinetic collision operator (15) contains FLR terms that have the form of a spatial diffusion. These terms correspond to the displacement of gyrocentres by distances $\sim \rho_{e}$ due to collisions during Larmor rotation. Solving the "zonal transport" problem more carefully, one can show that collisions also displace the centres of banana (and corresponding passing) orbits by distances of order the poloidal gyroradius $\rho_{p e}=\left(B / B_{p}\right) \rho_{e}$, which is larger. This leads to the damping rate (30). It is proprtional to $\nu_{e i}$ (rather than $\nu_{e e}$, on which it depends weakly) because it is essentially the Ohmic resistive damping of electron currents (both parallel and perpendicular; see Appendix F.4), and it is due to electron-ion friction (cf. [25]).

The calculation of Appendix F, where this is demonstrated more carefully, can be checked in 
our numerical simulations, to ascertain that it is indeed this effect that is responsible for the zonal damping. Figure 4 shows the zonal damping rate normalised by the collision frequency, $\gamma_{\mathrm{Z}} / \nu$, for a number of simulations in which the nonlinearity in equation (20) was turned off (the right-hand side set to zero) and the zonal field allowed to decay linearly. We see that, for a range of collisionalities $\nu$ and in a broad range of wave numbers $k_{x} \rho_{e}$, the scaling $(30), \gamma_{\mathrm{Z}} / \nu \sim k_{x}^{2} \rho_{p e}^{2}$, is followed quite well. As a further successful test, we find that if we turn off magnetic drifts $\left(\mathbf{v}_{B} \cdot \nabla h\right.$ in equation (20)), thus removing the banana orbits, the zonal damping rates drop by close to an order of magnitude (blue open circles in Figure 4). This is roughly consistent with a reduction of $\gamma_{\mathrm{Z}}$ by a factor of $\left(B / B_{p}\right)^{2} \approx(q R / r)^{2} \approx 18$ in our geometry, to $\gamma_{\mathrm{Z}} \sim \nu k_{x}^{2} \rho_{e}^{2}$, with the dominant diffusion due in this case to finite Larmor orbits, as explained above.

Finally, we test the theoretical expectation that the dominant contribution to the damping of the electron zonal flows, which are also currents, is proportional specifically to the electron-ion collision frequency $\nu_{e i}$. Figure 4 (black crosses) shows that when the electron-ion collisions are turned off, $\nu_{e i}=0$, leaving only the (momentum-conserving) electron-electron collisions $\nu_{e e}$, the damping rates drop dramatically and scale as $\gamma_{\mathrm{Z}} \sim \nu_{e e} k_{x}^{4} \rho_{p e}^{4}$, as indeed expected theoretically (see Appendix F.7).

Thus, the scaling (30) and the theory that leads to it (Appendix F) appear to be sound and successfully reproduced in our simulations.\|

\subsection{Numerical tests of the role of collisions and zonal modes}

With the theoretical argument presented in section 3.2 in mind, let us now build up the evidence that the long-time steady state of the ETG turbulence in our simulations is controlled by zonalnonzonal interactions and by the electron-ion collisional damping of the zonal modes. First, we remark that whilst linear simulations (see Appendix D) indicate that the linear instability growth rates may be comparable to the nominal (experimental) collisionality, the growth rates depend only weakly on collisionality. It does not seem plausible that such insensitive linear physics can explain the strong collisionality dependence of the nonlinearly saturated state. Clues to the actual (nonlinear) origin of this dependence can be obtained via nonlinear simulations with modified dynamics, as described below.

First let us show that the zonal component regulates the amplitude of the rest of the turbulence, which determines the heat flux. The cyan and purple curves in Figure 5 show the time evolution of the heat flux in simulations with identical parameters to one of the simulations in Figure 2, but with the nonlinear term artificially adjusted in such a way that the zonal modes no longer affect the evolution of the nonzonal modes: namely, the zonal components have been zeroed out in the calculation of the nonlinear term, so that in equation $(6),\left\langle\mathbf{v}_{E}\right\rangle \cdot \nabla h$ is replaced by $\left\langle\mathbf{v}_{E, \mathrm{NZ}}\right\rangle \cdot \nabla h_{\mathrm{NZ}}$. The zonal modes are still allowed to be nonlinearly driven by the nonzonal modes, but the zonal modes do not then feed back on the nonzonal modes; the nonzonal evolution is entirely independent of the zonal evolution. This eliminates the nonlinear terms used to obtain the dominant balance (24). The heat-flux collapse occuring in the full simulation is prevented by this change, confirming that the collapse is indeed mediated by the effect of the zonal modes (which was turned off) on the nonzonal modes (which carry the heat flux). The "quasi-saturated"

\| It is perhaps worth pointing out that such an agreement is only possible in simulations that use a sufficiently realistic electron gyrokinetic collision operator (see section 2.3), an indispensable property being momentum conservation by the electron-electron collisions and a correct capturing of Ohmic resistivity by the electron-ion ones. See, however, section 3.5 for certain simplifications that are allowed. 


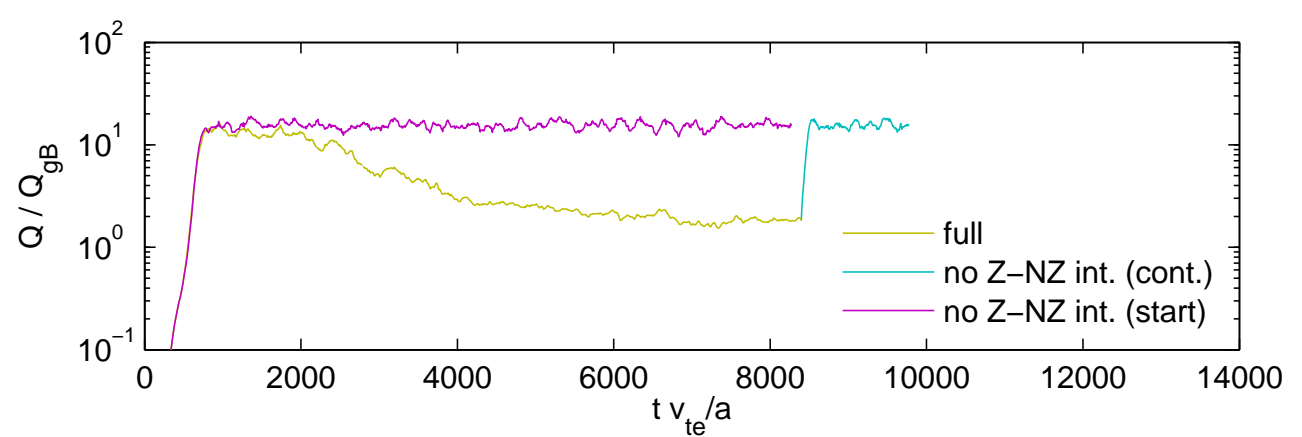

Figure 5. The large-box simulation shown in Figure 2 (yellow) was restarted at $t=$ $8397.7 \mathrm{a} / v_{t e}$ without zonal interactions in the nonzonal evolution equation (cyan). The heat flux in this modified simulation returns to a level that is close to the high early "quasisaturated" level. For direct comparison, the same modified simulation was also rerun from initial noise, giving the same heat flux level (purple).

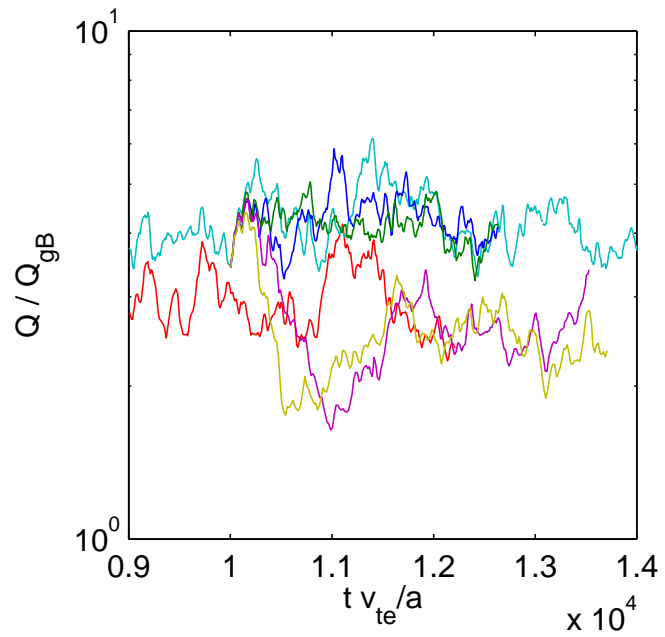

Figure 6. The two simulations corresponding to the red squares in Figure 3 are shown: the large-box simulations with $a / L_{T}=3.42$ and collisionalities $\nu=\nu_{\text {nom }} / 2($ cyan $)$ and $\nu=\nu_{\text {nom }} / 3$ (red). The higher-collisionality case is restarted at $t=10003.6 a / v_{t e}$ with certain terms in the collision operator reduced to the lower collisionality value, whilst others are retained at the higher value: (a) only electron-electron collisions reduced (blue); (b) only electron-ion collisions reduced (purple); (c) only nonzonal collisions reduced (green); (d) only zonal collisions reduced (yellow).

streamer-dominated state is thus just the saturated state that would have emerged had the zonal flows been prohibited or suppressed.

Collisions damp the zonal modes, and in this context are important precisely because they are small, as this means that the finite zonal fields that emerge from any fast collisionless evolution [24] are damped very weakly and so can grow to dynamically significant amplitudes and regulate 


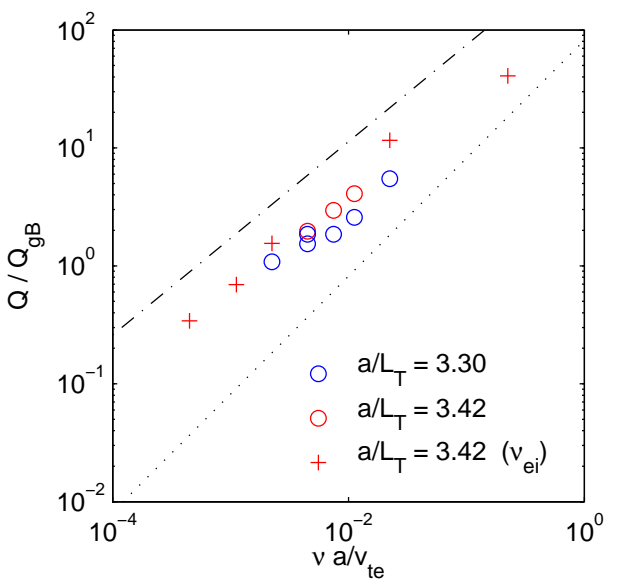

(a)

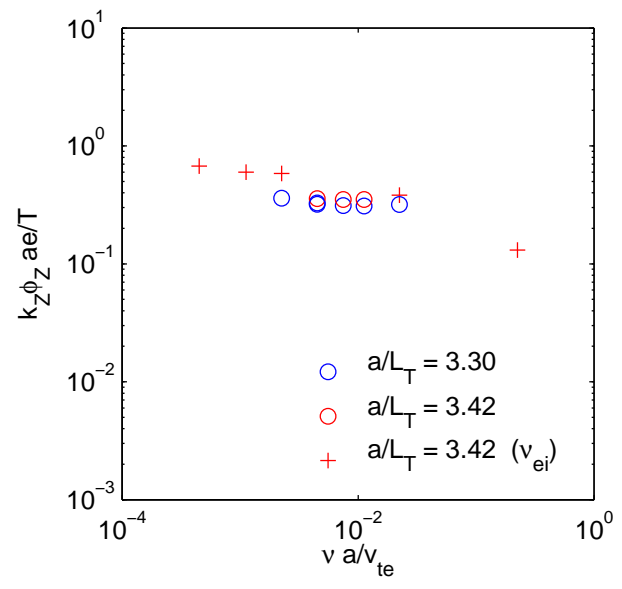

(b)

Figure 7. Variation of (a) the time-averaged electron heat flux $Q / Q_{\mathrm{gB}}$, (b) the rms zonal velocity $k_{\mathrm{Z}} \phi_{\mathrm{Z}}$, adding to the points from Figure 3 (now circles) further points (red crosses) obtained by varying only the electron-ion collisionality $\nu_{e i}$, but keeping the electron-electron collisionality at the nominal value, $\nu_{e e}=\nu_{\text {nom }}$, all at the nominal temperature gradient $a / L_{T}=3.42$. The dot-dashed line shows the experimental scaling (1) and the dotted line shows the theoretical linear scaling, $Q / Q_{\mathrm{gB}} \propto \nu_{*}$, equation (31).

the turbulence. Let us show that it is the electron-ion collisions that affect the zonal modes in the crucial way; these are momentum non-conserving (for electrons), as they act to relax the electron flow and thereby dissipate the associated current - in other words, they give rise to Ohmic resistivity (see discussion in section 2.3). In Figure 6, we show time evolution of the heat flux corresponding to two different collisionalities in the same series of simulations (the two shown as red squares in Figure 3 - the large-box simulations with $a / L_{T}=3.42$ ). The heat flux in the higher-collisionality simulation is shown by the cyan curve, the heat flux in the lower-collisionality one by the red curve. If we rerun the higher-collisionality simulation with $\nu_{e i}$ unchanged but $\nu_{e e}$ reduced to the lower value (blue curve), or with the zonal collisionality unchanged but the nonzonal collisionality reduced to the lower value (green curve), there is no significant change in the saturated heat flux. By contrast, if we reduce only $\nu_{e i}$, leaving $\nu_{e e}$ unchanged (purple curve), or if we reduce only the zonal collisionality, leaving the nonzonal collisionality unchanged (yellow curve), the heat flux drops to a value consistent with the lower-collisionality case (red). Thus it is the electron-ion collisions on the zonal component that primarily determine the heat-flux collisionality dependence.

\subsection{Simplified simulations for extended collisionality range}

We have argued that it is the effect of electron-ion collisions on the zonal modes that sets the collisionality dependence of the saturated heat flux. Collisions in the nonzonal gyrokinetic equation (19) are regularising, in that collisions are needed to dissipate the fine structure that the distribution function develops in velocity space. This means that, while collisions cannot be dropped for the nonzonal modes, the particular value of $\nu$ is unimportant or, at most, has a weak effect - for example on linear growth rates (see Appendix D). This is similar to the well-known situation in fluid turbulence, where a small viscosity is needed to provide dissipation but the 
saturated state is independent of the exact value of this viscosity. This understanding of the underlying physics opens up an opportunity to probe the collisionality dependence of electron transport without paying the high price of increased velocity-space resolution that reducing collisionality would exact. The strategy is to vary only the collisionality affecting the zonal modes or only $\nu_{e i}$ (although in the latter case, the subdominant part of the zonal damping rate $\sim \nu_{e e} k_{x}^{4} \rho_{e}^{4}$ — see section 3.3 and Appendix F.7 — will eventually take over).ף

Figure 7 shows the collisionality dependence of the electron heat flux over a wider range of values of $\nu_{e i}$ than in Figure 3, accessed using the latter strategy: varying $\nu_{e i}$ only while keeping $\nu_{e e}$ at its nominal value. We see that the result of this extended collisionality scan is to confirm the general plausibility of our picture of the ETG transport: $Q / Q_{\mathrm{gB}}$ stays approximately proportional to $\nu_{e i}$ across a wider range of its values, and the independence of the zonal flow velocity of $\nu_{e i}$ also approximately persists over this wider range. Note that these simulations were performed with reduced perpendicular spatial resolution and without flow shear; see Appendix C.2 and Appendix E for details.

\subsection{Spatial structure of the saturated state}

Finally, let us provide some details about the structure of the zonal and nonzonal components of the turbulence in its final saturated state.

The zonal component of the saturated turbulent field is long-lived, with a spatial structure that can persist, once established, for as long as the total simulation time. Figure 8 is a Hovmöller (space-time) diagram of the zonal potential for the same case as shown in Figure 2. The zonal pattern barely changes during the time window that we have used for time-averaging the heat flux and other quantities in the saturated state of this simulation. ${ }^{+}$

The spatial spectrum of the zonal potential is almost monochromatic in this particular case, as shown in Figure 10(a). In general, we find that the zonal spectra typically have only a small number of sharp peaks. A single extra harmonic is just visible in Figure 10(a).

By contrast with the monochromatic zonal spectrum, the nonzonal spectrum is broadband when averaged over time. Figure 10(c) shows the heat-flux spectrum against $k_{y}$, and Figure 11(a) shows the nonzonal $\phi$ spectrum for the large-box simulation of Figure 2 against both $k_{x}$ and $k_{y}$. Again by contrast with the static zonal component, the nonzonal component is rapidly fluctuating. Figures 11(b)-(d) show the same 2D spectrum of $\phi$ at particular instants of time. Thus, the nonzonal spectrum has a "flickering" appearance: at any given time, a small number of modes are much more intense than the others, but the dominant modes change over time, giving rise to a smooth time-averaged spectrum. This high "k-space intermittency" is perhaps natural in the

I We further stress that one should be careful in the interpretation of such simplified simulations outside the range of collisionalities used for the full simulations. The simplified simulations could become unrepresentative of the original system if the fixed electron-electron collisions suppress new modes that would otherwise have emerged and dominated the dynamics at either low or high collisionality. We have not investigated whether this is the case for the simulations reported here.

+ In this context, we gain some insight into the mechanism of generation of the zonal modes by returning to the numerical experiment shown in Figure 5 (section 3.4). We find that disconnecting the zonal feedback on the nonzonal modes but keeping the nonzonal-nonzonal interactions in the evolution equation for the zonal modes still leads to growth of the zonal modes in the "quasi-saturated" state, but they are quite different from the ones in simulations where their feedback is preserved: namely, they are more incoherent in time and they also grow more vigorously (see Figure 9). Thus, in the fully coupled system, even when the zonal modes are small, their excitation by the nonzonal modes still depends on the small modifications (subdominant as far as the heat flux is concerned) that they produce in the latter — and thus the mechanism of this excitation is not as straightforward as just stochastic coupling of nonzonal modes into $k_{y}=0$. 


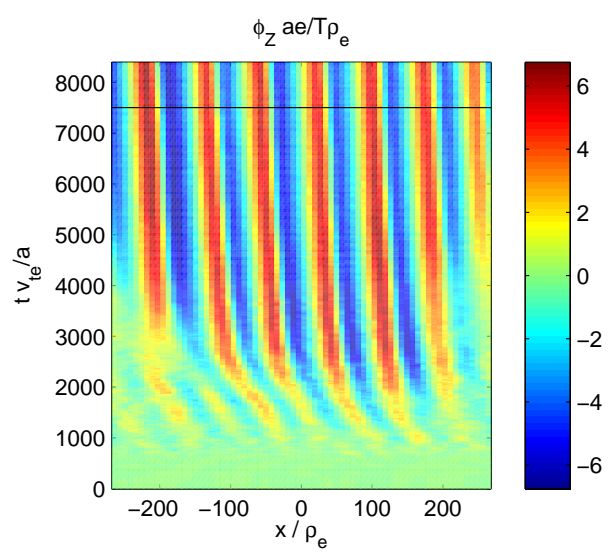

Figure 8. Zonal electrostatic potential $\phi_{\mathrm{Z}}$ in a flux-tube cross-section in the outboard midplane as a function of the radial spatial coordinate $x$ and time, for the same "large-box" simulation as shown in Figure $2\left(\nu=0.2 \nu_{\text {nom }}, a / L_{T}=3.3\right)$. The black line shows the start of the time-averaging window used to characterise the saturated state.

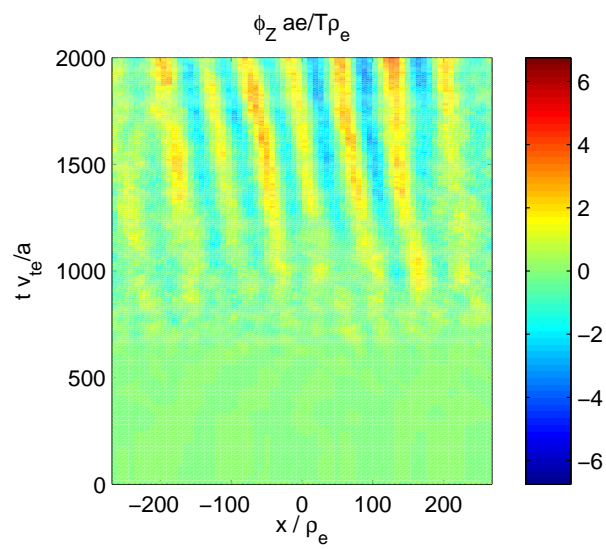

(a)

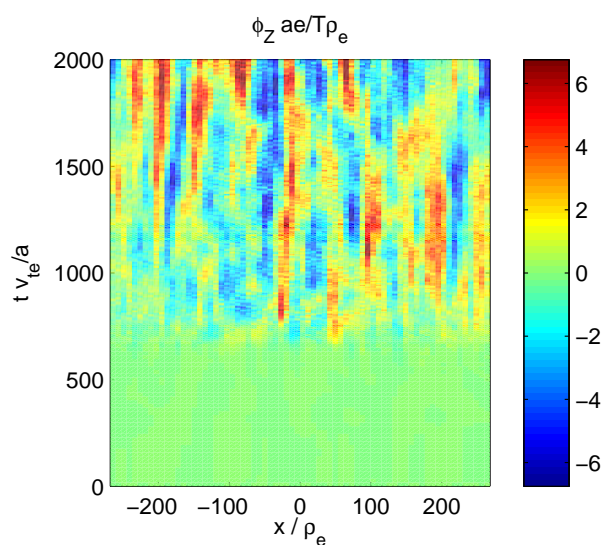

(b)

Figure 9. (a) The same as Figure 8, but for the initial period $t \leqslant 2000 a / v_{t e}$; (b) the same for the case in which zonal feedback onto the nonzonal modes has been disconnected (the simulation for which the heat flux is shown as the purple curve in Figure 5).

near-marginal saturated state at experimentally relevant parameters, with only a small number of modes excited at any given time. The details may be dependent on the simulation grid in $k_{x}$ and $k_{y}$, and this would be an interesting topic for future investigations.

\section{Summary and discussion}

The prevailing view of the structure of ETG turbulence in tokamaks and the associated levels of transport has its origin in the first gyrokinetic simulations, which did not include collisions [10, 11] (and did not correspond to near-threshold conditions in a spherical tokamak). Whereas the ITG turbulent state has long been believed to be zonal-flow dominated [26], the ETG fluctuations 


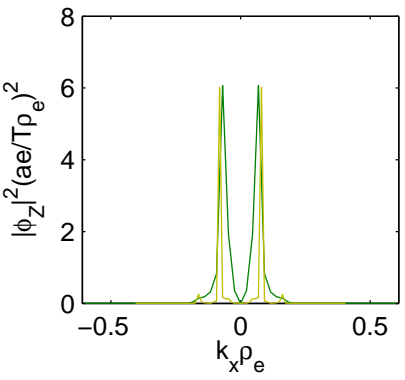

(a)

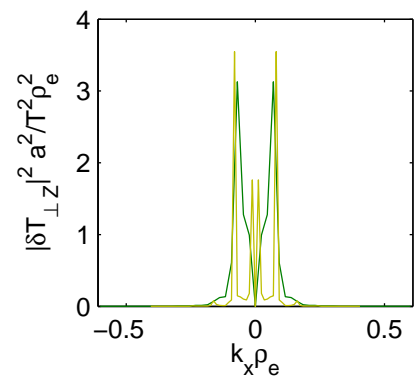

(b)

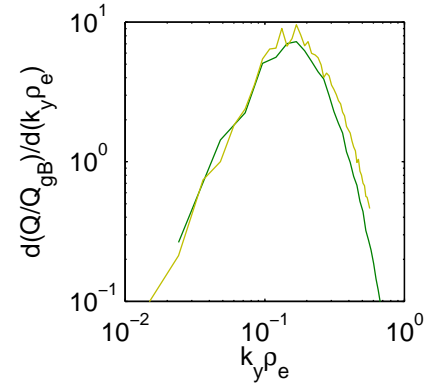

(c)

Figure 10. For the same case as Figure 2: (a) the spectrum of the zonal potential; (b) the spectrum of the zonal perpendicular temperature perturbation; (c) the heat-flux spectrum; all are averaged over the time window of the saturated state, which is shorter for the large-box simulation (yellow) than for the small-box simulation (green).

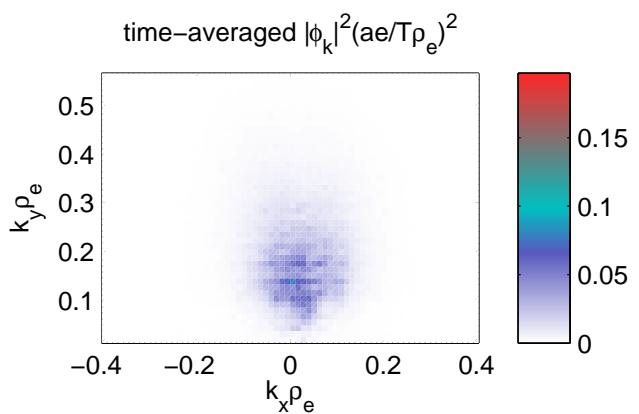

(a)

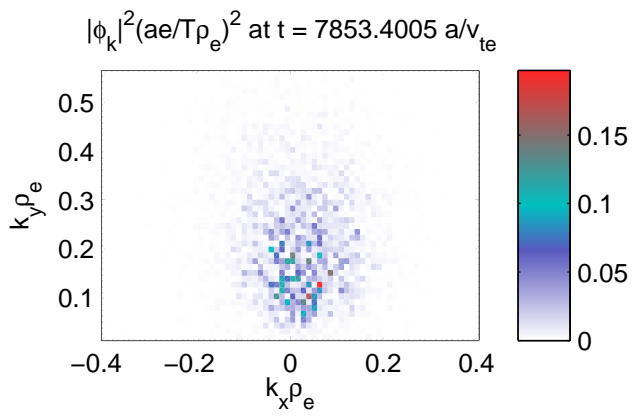

(c)

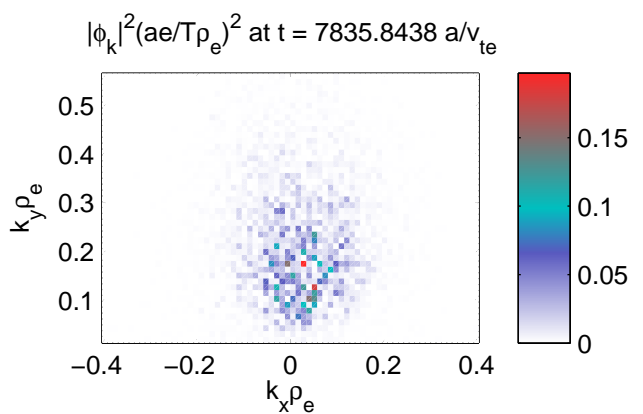

(b)

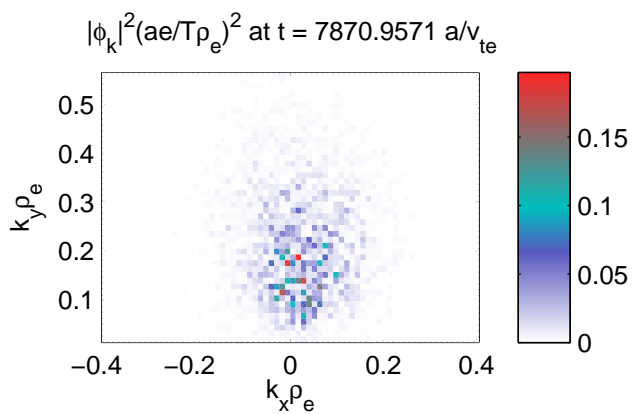

(d)

Figure 11. 2D spectra of the nonzonal electrostatic potential for the same large-box simulation as in Figure 2: (a) the spectrum averaged over the saturated time-window $t \geqslant 7502.3 \mathrm{a} / \mathrm{v}_{\text {te }}$; (b) instantaneous spectrum at the same time as Figure 1(b); (c) \& (d) instantaneous spectra at later times during the saturated state. The grid-cell size in these plots is 0.012 in both directions: the minimum positive $k_{x} \rho_{e}$ and $k_{y} \rho_{e}$ (see Appendix C.2).

were characterised by long radial eddies ("streamers") that enhanced the transport to a level comparable with ITG turbulence, overcoming the reduction by a factor of $\left(m_{e} / m_{i}\right)^{1 / 2}$ expected from the relationship between the electron and ion gyroscales (the scales at which the two types of fluctuations were driven). 
The present study differs from the more traditional approach to modelling ETG turbulence in three respects: collisions are included; simulations are run for a much longer time; and the equilibrium parameters correspond to the experimental situation in a real device, namely MAST, and therefore place the system close to a marginal state with respect to the ETG drive (note also that MAST is a spherical tokamak, so has a somewhat different magnetic geometry compared to the more prevalent large fusion devices such as TFTR, JET or ITER).

As a result, we find that, in application to the physical regime that we have considered, the standard picture of ETG transport is in need of revision. The high-transport, streamer-dominated nonlinear state does indeed emerge, and is not strongly dependent on the collisionality of the plasma, but it persists only transiently, over relatively short simulation times (short compared to the energy confinement time but still long compared to a typical eddy turnover time, and long compared to typical simulation times used for ETG turbulence in the past). It turns out that this state is not entirely steady — while the heat flux might appear to be statistically stationary, there is a slow growth of the zonal component of the fluctuations, which eventually (after $t v_{t e} / a \sim$ a few thousand) reaches dynamical strength compared to the transport-setting nonzonal modes and proceeds to change the character of the turbulence. A new, long-time, zonaldominated saturated state emerges, whose structure is more reminiscent of what is traditionally expected of ITG, rather than ETG, turbulence (see Figure 1 and further discussion in section 4.2). We emphasize that it is the final saturation level of the heat flux in gyrokinetic flux-tube simulations, averaged over the turbulent fluctuation scales in length and time, that is physically relevant for transport calculations. We have found that the turbulent heat flux supported by the new long-time nonlinear state can be much lower than in the "quasi-saturated" streamerdominated state if the collisionality of the plasma is low (see Figures 2(a), 3(a) and 7(a)). The (roughly linear) collisionality dependence of the heat flux found in our simulations turns out to be in remarkably good agreement with the experimental scaling (1) [1].†

We have proposed a phenomenological argument (section 3.2) whereby this collisionality scaling can be understood if one assumes that the saturation of the nonzonal modes is governed by the zonal gradients coming into approximate balance with the equilibrium gradients (to be more precise, the zonal-nonzonal interactions balancing the linear drive), while the saturation of the zonal modes is set by a balance between their nonlinear excitation by the nonzonal interactions and their damping by Ohmic resistivity. The latter is operative because electron-scale flows are also currents - and so it is the electron-ion collisions that play the defining role in setting the zonal damping rate. We have supported our view by a series of numerical experiments that confirmed the crucial role of the zonal modes in enabling the emergence of the new saturated state (section 3.4) and the crucial role of the electron-ion collisions on the zonal modes in setting its collisionality dependence (sections 3.4 and 3.5). The Ohmic damping of the zonal flows is an analytical result (Appendix F), but we have also systematically confirmed that it is captured correctly in our simulations (section 3.3). We have also documented some key qualitative features of the long-time saturated state: the long-time coherence and approximate monochromaticity of the zonal modes (cf. [29, 30]) and, in contrast, the dominant individual nonzonal modes "flickering" with time in and out of existence to give rise to a broad-band time-averaged spectrum (section 3.6).

$\dagger$ Earlier simulations of ETG in MAST [27, 28] did report heat fluxes roughly consistent with the estimated experimental level of electron heat transport in the cases that were simulated. Whilst we obtain a difference in heat flux between the "quasi-saturated" state and the final state across the entire range of collisionality shown in Figure 3, including at the nominal collisionality, the most dramatic differences occur when the collisionality is well below its nominal value (see Figure E2(a)). 


\subsection{Previous work on ETG and the collisionality scaling}

Long-time changes in the saturated state of gyrokinetic simulations have previously been reported in other numerical studies. Mantica et al. [31] reported a change in the zonal-nonzonal balance at long times in an ITG simulation. Guttenfelder and Candy [32], in their collisionless ETG simulations with adiabatic ions using the GYRO code, found a long-time reduction in transport associated with an increased level of zonal perturbations, which occurred at a low but not at a higher level of flow shear. We have not investigated here the dependence on flow shear, except via the simplified simulations with zero flow shear reported in section 3.5, which appear to obey the same scalings (Figure 7). It is not impossible that the transport scaling found in the present paper exists within a window in which the flow shear is not too large (although it should perhaps still be large enough to suppress ion-scale transport in order for a heat-flux calculation restricted to electron scales to make sense).

Perhaps most relevantly for comparisons with our work, Nakata et al. [33], using a slab ETG model, adiabatic ions, a Lorentz collision operator (i.e., the pitch-angle scattering operator in (15)), and comparing two different sets of parameters, reported that transport at long times in their linearly more unstable case could be suppressed below the level of their linearly more stable case, owing to the formation of zonal flows that collimated the turbulence into "vortex streets" and acted as a barrier to radial transport (see also earlier studies of ETG zonal flows [34, 35]). This suppression appears to be consistent with our findings.

Recent direct measurements using the Doppler back-scattering system installed on MAST [36] are consistent with small-scale turbulence due to ETG. Gurchenko and Gusakov [37] established an experimental correlation in several devices between electron-scale turbulence and anomalous electron transport, but not a causation pathway directly attributing the transport to ETG. The transport scaling that we obtain here derives from, and may, therefore, be an experimental signature for, not only the presence of electron-scale turbulence but also the saturation mechanism involving a dominant interaction with weakly damped zonal modes. In a regime in which this were not the saturation mechanism, the scaling of transport with collisionality may well be much weaker or absent.

An alternative explanation for the experimental collisionality scaling of heat transport in spherical tokamaks that has been previously suggested relies on the transport associated with microtearing turbulence [1, 38, 39]. Since, in the present work, we have limited ourselves to electrostatic perturbations, the microtearing instability is excluded and and so it is clear that the collisionality scaling that we have found does not require it. Note that the arguments we apply to ETG-driven turbulence are fairly generic and may well apply in some form to other instabilities. We have, of course, also not excluded the possibility that other modes may produce a similar dependence for different reasons. The microtearing contribution in particular to the overall electron transport in real fusion devices remains a live and pressing research subject (made challenging, however, by the particular difficulty of obtaining well-resolved simulations of electromagnetic turbulence in tokamaks).

It is an interesting question how general our picture of ETG turbulent transport might prove to be. NSTX [40] and MAST [1] both exhibit the strong scaling of confinement time with collisionality considered here, but in conventional tokamaks (as opposed to these two spherical ones), the scaling exponent between $B \tau_{E}$ and $\nu_{*}$ has been found to be much weaker or essentially zero [41, 42, 43]. Clearly, identifying the reason or reasons for this difference is an important issue for further study. We expect ETG to be more important in STs for the overall energy confinement, because of the large flow shear available to suppress ion-scale modes. 
Considering just ETG-dominated electron heat transport, however, one possibility is that, since in conventional tokamaks $B / B_{p}$ is large compared to STs, giving larger zonal damping, the heat flux given by the scaling (31) may be as large as the heat flux in our "quasi-saturated" state, in which case the "quasi-saturated" state may be the actual saturated state, not regulated by the mechanism discussed here, and with only weak or no collisionality dependence. $\ddagger$

\subsection{ETG vs. ITG turbulence near and far from threshold}

The idea that the ETG turbulent state is dominated by zonal modes leads one naturally to the question of whether the saturated states of ETG and ITG turbulence are essentially similar, at least qualitatively.

Our ETG turbulence model, equations (6) and (14), describes kinetic electrons with Boltzmann (adiabatic) ions. The simplest possible model for ITG turbulence would involve kinetic ions with Boltzmann electrons - the latter physically justified by fast streaming of electrons along field lines. The main mathematical difference between these two models is that the Boltzmann electron response must be restricted to perturbations that have variation along magnetic-field lines. Namely, the density perturbation is, in contrast to the ETG case (13),

$$
\frac{\delta n}{n}=\frac{e(\phi-\bar{\phi})}{T_{e}},
$$

where $\bar{\phi}$ is the flux-surface average $[44,45,46]$. It is this latter feature that is normally believed to be responsible for the difference between the zonal-flow-dominated ITG state and the streamerdominated ETG state: radial variations in the zonal $\phi$ do not affect $\delta n$ as electrons cannot respond to radially varying zonal modes at ion scales; these can, therefore, grow large enough to break up the primary ITG-driven streamers via a secondary instability [47, 23] and isotropise the turbulence, whereas for ETG turbulence, the latter effect was believed to be too weak to destroy the streamers $[10,11]$ (although the physics that determined the radial scale of the streamers perhaps remained unclear [48] — possibly again a (weaker) secondary instability [47, 49]).

What we have found in our simulations is that, whereas there is indeed no trace of a fast onset of a secondary instability similar to one that breaks up the ITG streamers (and is indeed seen in most ITG simulations), the ETG streamers' lease on life granted by a stronger density response to zonal perturbations is nevertheless only temporary: the zonal component does find a way to grow slowly, until it is large enough to take control over the nonzonal fluctuations.

In addition to the ITG instability giving rise to streamer-like modes and the secondary instability breaking them up into zonal flows, the third pillar of the standard picture of ITG saturation is a "tertiary instability" whereby the zonal modes that have grown to a certain critical amplitude break up, returning energy to nonzonal perturbations [23]. This mechanism of regulating the zonal component of the turbulence does not rely on collisional damping and thus should give rise to heat fluxes that are mostly independent of collisionality - as indeed it appears to do, at least when the ITG turbulence is simulated in regimes that are far from the instability threshold [20]. However, it has long been known that at temperature gradients that are close to the linear threshold (and thus, arguably, most relevant experimentally), the tertiary instability is ineffective,

$\ddagger$ More generally, something similar may happen in ranges of collisionality, background gradients or other aspects of the magnetic geometry that differ from those considered here. However, even in conventional tokamaks, the collisionality scaling from the argument that we have presented may still be recovered at sufficiently low values of collisionality. Testing this hypothesis in simulations of conventional tokamaks would be an interesting avenue to explore in the future. 
and a state of strongly suppressed transport ensues, called the Dimits upshift of the critical temperature gradient [50,23]. Clearly, in the case of ETG turbulence, should a tertiary instability of the zonal modes appear, their collisional damping would cease to matter and consequently the collisionality dependence of the heat flux should flatten off (cf. [51]). It is then tempting to suppose that our ETG state, in which the zonal modes are stable, collisionally damped and the heat flux scales with collisionality, is simply the ETG version of the Dimits-shift regime. Ascertaining whether this is indeed the case requires a larger parameter scan (in particular, in the temperature gradients) than has been undertaken here and has to be left for future work. By analogy with the ETG case, another tempting conjecture would be that the Dimits regime for the ITG turbulence should also have an (ion) collisionality scaling, by way of an argument similar to one presented in section 3.2. This in fact is not a new idea [52, 53, 26, 51], although the situation remains murky as including more realism (in particular, kinetic electron response) in gyrokinetic simulations of ITG turbulent transport can nullify or even reverse its collisionality dependence [54]. Since the collisionality scaling of the ETG transport that we report at least has some experimental support [1], we feel more secure about the relevance of our conclusions — although "stress-testing" them by relaxing various assumptions (e.g., perhaps most urgently, allowing kinetic ions [48]) and by extending the equilibrium parameter ranges is an essential direction for future research.

Thus, the relationship between ETG and ITG is complicated and indeed within each of these two regimes, there are likely to be several sub-regimes depending on the part of the parameter space that one is interested in - in particular, how far from the threshold the turbulence is. This said, our results suggest that there are perhaps more similarities between ETG and ITG physics than previously thought. Seeking a unified and universal description of various types of drift-wave turbulence may therefore be a worthwhile and reasonable aspiration.

In conclusion, let us summarise again what has been achieved here. We have found that in the saturated state of ETG turbulence at driving gradients close to their experimental values in MAST, the electron heat transport decreases with collisionality, in agreement with experimental evidence [1]. This behaviour points toward improved confinement in future devices. We have explained it based on a simple theoretical picture (backed up by numerical tests) of an ETG turbulent state dominated by the interaction between nonzonal and zonal modes, with the collisionality dependence of the heat flux originating from the collisionality dependence of the resistive damping of the zonal modes. On a practical note, ETG simulations with generic initial conditions must be run to very long times to capture the (crucial) effect of zonal modes on the saturated state.

\section{Acknowledgments}

This work was supported in part by EPSRC under grant numbers EP/H002081/1, EP/I501045/1, $\mathrm{EP} / \mathrm{L} 000237 / 1$ and EP/M022463/1; and by the EC under grant agreement number 633053 .

$\S$ In porting this argument to the ion scales, one must not forget that an important difference between the ETG and ITG cases is the specially pivotal role played by electron-ion collisions for the ETG zonal modes. For ions, ion-electron collisions are always subdominant in the mass-ratio expansion because the ions carry nearly all of the momentum, and collisions with the lighter electrons have a negligible impact on the ion flow. By contrast, the relative flow of electrons (which constitutes a current) is strongly affected by collisions with the heavier, sluggish ions. Therefore, for electrons, collisions are not momentum-conserving, whereas for ions, to lowest approximation they are. How this difference plays out in the calculation of the rate of damping of ion zonal flows is outlined in Appendix F.8. In the end, it turns out, however, that the damping rate scales in the same way as for the electron case, $\gamma_{\mathrm{Z} i} \sim \nu_{i i} k_{x}^{2} \rho_{p i}^{2}$, although for a different reason. 
The work of AAS was also supported in part by grants from the UK STFC and EPSRC. YcG was supported by the National R\&D Program through the National Research Foundation of Korea (grant number NRF-2014M1A7A1A01029835), and the KUSTAR-KAIST Institute. WD was supported by the US DOE grants DE-FC02-08ER54964 and DE-FG02-93ER54197. Computations were performed at the UK's HECToR and ARCHER services, on EFDA's HPCFF facility, and on the Helios system at IFERC-CSC. The authors thank Ian Abel, Jack Connor, Steve Cowley, Greg Hammett, Edmund Highcock, Walter Guttenfelder and Ferdinand van Wyk for enlightening discussions; and also thank Justin Ball, Francis Casson, Paul Dellar, Anthony Field, Jon Hillesheim, Nuno Loureiro, Martin O'Brien, Martin Valovič and two anonymous referees for helpful comments on versions of this paper. We are grateful to the Wolfgang Pauli Institute, Vienna, for its hospitality on several occasions.

\section{Appendix A. Correspondence between $B \tau_{E}$ and $Q / Q_{\mathrm{gB}}$ scalings}

In the experimental scaling (1), $\tau_{E}$ is the energy confinement time, i.e., the time it takes the power loss $P \sim Q A$ to carry away the thermal energy $W \sim n T V$, where $A$ and $V$ scale with flux surface area and volume respectively, $Q$ is the energy flux, $n$ is density, and $T$ is temperature. Therefore,

$$
\tau_{E} \sim \frac{W}{P} \sim \frac{n T a}{Q},
$$

where $a \sim V / A$ is the macroscopic length scale.

The appropriate normalising quantity for $Q$ is the "gyro-Bohm" heat flux $Q_{\mathrm{gB}}=n T v_{t e} \rho_{*}^{2}$, where $v_{t e}=(2 T / m)^{1 / 2}$ is the thermal speed, $\rho_{*}=\rho_{e} / a, \rho_{e}=v_{t e} / \Omega_{e}$ is the gyroradius and $\Omega_{e}$ is the cyclotron frequency (since we are interested in electron transport, we are using electron quantities, but the argument for ions is exactly the same). In the gyrokinetic ordering, the heat flux is $Q=\mathcal{O}\left(\rho_{*}^{2}\right)$ automatically (see, e.g., [14]), so the normalised heat flux $Q / Q_{\mathrm{gB}}$ must be order unity and independent of $\rho_{*}$. This normalised heat flux is

$$
\frac{Q}{Q_{\mathrm{gB}}}=\frac{Q}{n T v_{t e} \rho_{*}^{2}}=\frac{Q}{n T a \Omega_{e} \rho_{*}^{3}} \sim \frac{1}{\Omega_{e} \tau_{E} \rho_{*}^{3}},
$$

where we have used (A.1) to obtain the last expression. At constant $\rho_{*}$, (A.2) implies

$$
\frac{Q}{Q_{\mathrm{gB}}} \propto \frac{1}{B \tau_{E}}
$$

so we are probing the same dependence on collisionality by measuring $Q / Q_{\mathrm{gB}}$ in gyrokinetic simulations as the experiments do by measuring $B \tau_{E}$ with $\rho_{*}$ fixed.

Let us now explain what is meant when scaling (1) is claimed to hold experimentally [1]. The starting point is to assume that the following parametrisation of the energy confinement time holds:

$$
\Omega_{e} \tau_{E}=K \nu_{*}^{x_{\nu}} \rho_{*}^{x_{\rho}} q^{x_{q}} \beta^{x_{\beta}} \kappa^{x_{\kappa}},
$$

where $K$ is a constant, $\kappa$ is elongation [55], $\beta$ is plasma beta, $q$ is the safety factor and

$$
\nu_{*}=\frac{\nu}{\varepsilon^{3 / 2} v_{t e} / q R}
$$


is the effective electron collision rate $\nu / \varepsilon$ divided by the bounce frequency $\varepsilon^{1 / 2} v_{t e} / q R$, where $\varepsilon=a / R$ is the inverse aspect ratio, and $R$ the major radius. This is the dimensionless quantity characterising collisional detrapping. The experiments looking for the "collisionality scaling" strive to hold all these parameters constant apart from $\nu_{*}$ and measure the scaling exponent $x_{\nu}$ of $B \tau_{E}$ with respect to $\nu_{*}$. Since in our numerical study, we do not vary the equilibrium geometry, the same exponent $x_{\nu}$ will apply to the normalised collisionality in our gyrokinetic simulations, which is simply $\nu a / v_{t e}$.

Note finally that, in view of (A.2), if $Q / Q_{\mathrm{gB}}$ is independent of $\rho_{*}$ (as it is hard-wired to be within the gyrokinetic ordering), it must be the case in (A.4) that $x_{\rho}=-3$, which is indeed consistent with the experimental findings [1].

\section{Appendix B. Plasma parameters}

The reference, or "nominal", plasma parameters for our simulations represent MAST shot 8500 at $t=0.289 \mathrm{~s}$. They are specified in Table B1. We use a Miller [55] representation of the flux-surface geometry, which is assumed to be axisymmetric.

\begin{tabular}{lcc}
\hline minor radius & $r / a$ & 0.65 \\
major radius & $R / a$ & 1.46 \\
safety factor & $q$ & 1.9 \\
magnetic shear & $\hat{s}$ & 1.8 \\
Shafranov shift & $R^{\prime} / a$ & -0.25 \\
pressure gradient & $p^{\prime} \beta / p$ & -0.12 \\
elongation & $\kappa$ & 1.57 \\
elongation gradient & $\kappa^{\prime}$ & 0.40 \\
triangularity & $\delta$ & 0.22 \\
triangularity gradient & $\delta^{\prime}$ & 0.16 \\
\hline density gradient & $a / L_{n}$ & -1.2 \\
electron temperature gradient & $a / L_{T}$ & 3.42 \\
effective ion charge & $Z_{\text {eff }}$ & 1.15 \\
species temperature ratio & $T_{e} / T_{i}=1 / \tau$ & 1.06 \\
electron collisionality & $\nu_{\text {nom }} a / v_{t e}$ & 0.02 \\
flow shear & $\gamma_{E} a / v_{t e}$ & -0.003 \\
\hline
\end{tabular}

Table B1. Nominal dimensionless local equilibrium parameters based on MAST shot 8500 at $t=0.289 \mathrm{~s}$. Here $a=0.55 \mathrm{~m}$ is the half diameter of the last closed flux surface at the elevation of the magnetic axis and $r$ is the same for the particular flux surface we have chosen. The prime symbol denotes the derivative with respect to normalised minor radius: ' $\equiv a(d / d r)$. Scale lengths are defined by $a / L_{n} \equiv-n^{\prime} / n$ (density) and $a / L_{T} \equiv-T^{\prime} / T$ (temperature).

Note that the reversed density gradient is not rare in MAST [56] and was found to be linearly stabilizing [57].

In our simulations, we varied the collisionality $\nu$ relative to its nominal value $\nu_{\text {nom }}$ given in Table B1. We carried out such a collisionality scan for two values of $a / L_{T}$, the nominal one given in Table B1 and a slightly smaller (slightly more marginal) $a / L_{T}=3.3$. All other parameters were held fixed.

A small amount of flow shear $\gamma_{E}$, given in Table B1, was included in the nonlinear simulations. As in [28], this was found to assist convergence and is of the same order as the experimental 
level in MAST (see Appendix E.2). The sign of $\gamma_{E}$ is unimportant because the equilibrium is up-down symmetric [58].

\section{Appendix C. Numerical details}

\section{Appendix C.1. GS2 spatial coordinates}

GS2 solves the gyrokinetic equation (6) in a flux tube that winds its way toroidally and poloidally around the toroidal flux surface designated by a label $r$ (see Table B1), which is defined as its half diameter at the elevation of the magnetic axis of the tokamak. The position along the flux tube is determined by the poloidal angle $\theta$, which can be thought of as the coordinate parallel to the magnetic field. The flux-tube domain that we use in GS2 extends over $-\pi \leq \theta \leq \pi$, where $\theta=0$ is the outboard midplane and $\theta= \pm \pi$ the inboard midplane.

If we demand that

$$
\mathbf{B}=\nabla \alpha \times \nabla \psi
$$

where $\psi$ is the poloidal magnetic flux and $\alpha$ is chosen to satisfy (C.1), each field line is labelled by the pair of Clebsch coordinates $(\psi, \alpha)$. Then $(\psi, \alpha, \theta)$ can be used as the three independent (but not orthogonal!) curvilinear coordinates. Designating the central field line of a flux tube by $\left(\psi_{0}, \alpha_{0}\right)$, the GS2 coordinates $(x, y)$ transverse to the magnetic-field direction are related to $(\psi, \alpha)$ in the following manner $[59,60]$ :

$$
\begin{aligned}
& x=\frac{q\left(\psi_{0}\right)}{B_{0} r\left(\psi_{0}\right)}\left(\psi-\psi_{0}\right), \\
& y=\left.\frac{1}{B_{0}} \frac{d \psi}{d r}\right|_{r=r\left(\psi_{0}\right)}\left(\alpha-\alpha_{0}\right),
\end{aligned}
$$

where the safety factor $q(\psi)$ and the radial flux-surface label $r(\psi)$ are both flux functions and $B_{0}$ is a reference (constant) magnetic field defined to be the toroidal magnetic field at the midradius of the flux surface containing our flux tube. The $x$ coordinate is "radial" in the sense that it is transverse to the flux surface and increases away from the magnetic axis. The second coordinate $y$ is often called "binormal" because it effectively measures distances perpendicular to the field line but within the flux surface (at constant $\psi$ ). In the outboard midplane, $(x, y)$ form an approximately orthogonal grid in the cross-section of the flux tube perpendicular to the magnetic field, with $x$ and $y$ approximately measuring true distances from the centre of the flux tube. Following the field line around the flux surface, the flux tube twists due to magnetic shear, with $\nabla x, \nabla y$ and $\nabla \theta$ generally not orthogonal to each other.

GS2 is pseudospectral with respect to $(x, y)$ but not $\theta$; that is, except for the evaluation of the nonlinear term, its data grid has the coordinates $\left(k_{x}, k_{y}\right)=\mathbf{k}_{\perp}$ and $\theta$. For the evaluation of the nonlinear term, the Fast Fourier Transform is used to go from $\left(k_{x}, k_{y}\right)$ to $(x, y)$ (and then back again after the evaluation). In the context of the GS2 grids, the terms "parallel" and "perpendicular" refer to $\theta$ and to the other two coordinates (either $(x, y)$ or $\left(k_{x}, k_{y}\right)$ as appropriate), respectively.

\section{Appendix C.2. Numerical grids, resolution and boundary conditions}

In our largest nonlinear simulations, the spatial grid has 48 cells in $\theta$ and $108 \times 144$ points in $x \times y$, the range of positive perpendicular wavenumbers being $\left(k_{x} \rho_{e}, k_{y} \rho_{e}\right) \in[0.012,0.40] \times[0.012,0.57]$. 
We refer to these as "large-box simulations" in the text. The simulations referred to as "smallbox simulations" are the same except for the perpendicular grid parameters: the box size in real space is halved, but extended to somewhat higher perpendicular wavenumbers (i.e., higher spatial resolution): $80 \times 108$ in $x \times y$, spanning the spectral range $\left(k_{x} \rho_{e}, k_{y} \rho_{e}\right) \in[0.023,0.60] \times[0.024,0.84]$. The parameter regimes in which these two classes of simulations are useful are discussed in Appendix E. The wavenumber ranges are intended to capture electron physics; the Boltzmann ion response ensures that we need not worry about where they lie in relation to ion scales.

Dealiasing is used for the nonlinear term: zero-padding with higher-wavenumber modes is introduced before the Fourier step into real space; any nonlinear interactions that wrap around unphysically in reciprocal space will be nullified when these modes are thrown away after the inverse Fourier step. The number of $x \times y$ points indicated here (applicable only to nonlinear runs) is the same as the total number of modes including the dealiasing modes, but the ranges of wavenumbers given above do not include the dealiasing modes. All data shown here, including plots in real space, derive solely from the evolved, non-dealiasing modes: the additional dealiasing modes are used only transiently during the nonlinear part of each time step, and are not included in the code's output.

Twist-and-shift parallel boundary conditions $[61,59]$ link together some of the $k_{x}$ modes at low $k_{y}$, whereas the $k_{y}=0$ modes are periodic in $\theta$. At the remaining (open) domain ends, the parallel boundary condition is $h=0$ for incoming particles. The boundary conditions are automatically periodic in the perpendicular directions $(x, y)$ by virtue of the spectral representation.

The velocity-space grid has 18 energies $\mathcal{E}=v^{2} / 2$, and, for passing particles, 16 grid-points in $\lambda=\mu / \mathcal{E}$ for each sign of $v_{\|}= \pm(2 \mathcal{E}-2 \mu B)^{1 / 2}$, where $\mu=v_{\perp}^{2} / 2 B$ is the magnetic moment. Trapped particles are accommodated by additional $\lambda$ grid points, one for particles bouncing at each $\theta$.

Linear simulations (Appendix D) use the same setup as nonlinear ones, but evolve each perpendicular wavenumber independently.

For the "simplified" simulations in section 3.5, in which only the electron-ion collisionality was varied, the grid sizes were: 24 cells in $\theta ; 40 \times 54$ points in $x \times y$, spanning the spectral range $\left(k_{x} \rho_{e}, k_{y} \rho_{e}\right) \in[0.023,0.30] \times[0.024,0.41] ; 12$ energies $\mathcal{E}$; and $8 \lambda$ 's for passing particles in each direction. This version of the code applied the zero incoming boundary condition to $g=\langle\delta f\rangle=h+e\langle\phi\rangle F / T$, rather than to $h . \|$

\section{Appendix D. Linear growth rates}

Figure D1 shows the growth rate of the most unstable linear mode at $k_{x}=0$ for each $k_{y}$ in the numerical domain (which was described in Appendix C.2). These linear simulations have zero flow shear, $\gamma_{E}=0$.

At the given $a / L_{T}$, the effect of decreasing collisionality is to decrease slightly the maximum growth rate, and to extend to somewhat lower $k_{y}$ the unstable wavenumber interval. At $k_{y} \rho_{t e} \approx 0.2$ where the saturated heat flux is maximal (see Figure 10(c)), the dependence of the linear spectrum on collisionality is weak.

\| The option to use $h$ was added to the code during the course of the present work. The difference between the two options vanishes as $\phi \rightarrow 0$ at $\theta \rightarrow \pm \infty$, but it has been found in linear simulations [62] that the use of $h$ leads to faster convergence as the actual, finite $\theta$ domain size is increased. 


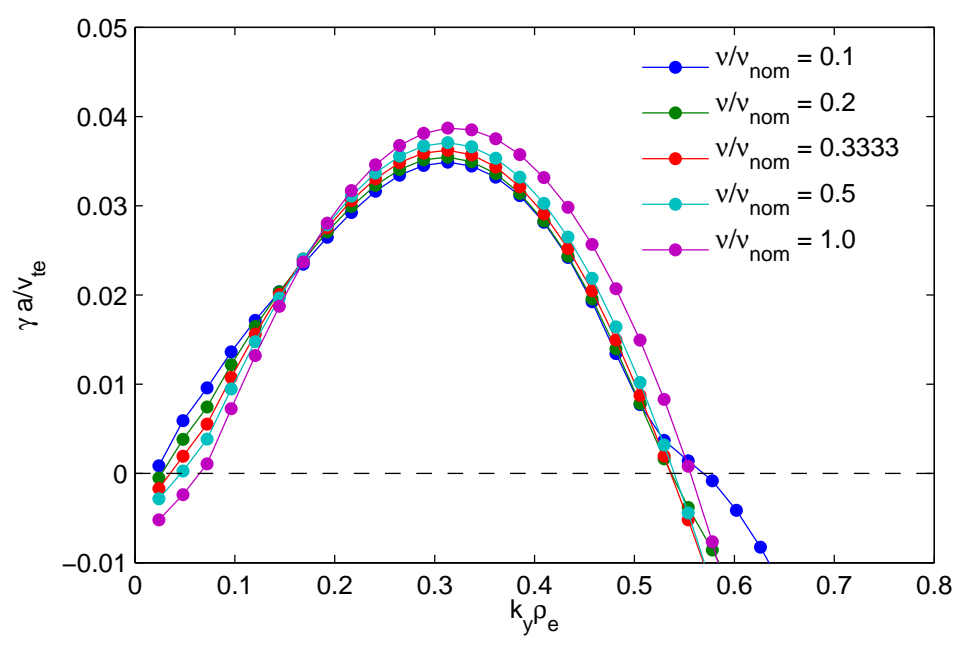

Figure D1. Linear growth rates versus $k_{y}$, at $k_{x}=0$, with zero flow shear $\gamma_{E}=0$, for various collisionalities as indicated. All other parameters are the nominal ones given in Table B1 (including $a / L_{T}=3.42$ ).

Note that this scan is consistent with the previous observation [28] that collisions lead to a stability gap between ETG- and ITG-driven modes (the range of $k_{y}$ 's unstable to ITG being lower than the wave numbers shown here).

Figure D2 shows the dependence of the maximum growth rate on the temperature gradient (still with $\gamma_{E}=0$ ). We see that the linear critical gradient varies only between 2.3 and 2.5 throughout this whole range spanning a factor of 50 in collisionality. This is in agreement with the earlier findings [63] that collisions have very little effect on the linear critical gradient.

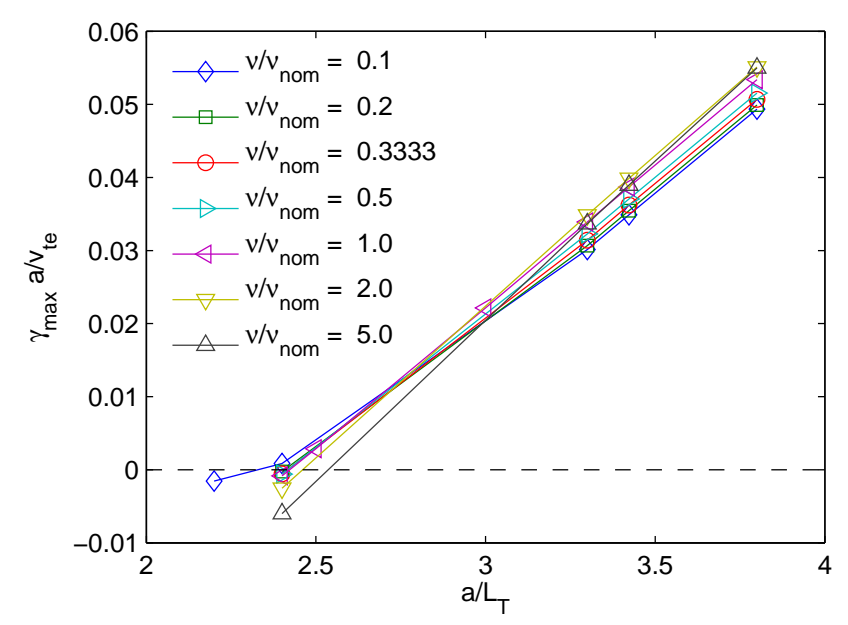

Figure D2. Linear growth rates versus $a / L_{T}$, with zero flow shear $\gamma_{E}=0$, for various collisionalities as indicated, showing that the linear critical gradient (where the growth rate crosses zero) is insensitive to collisionality. 


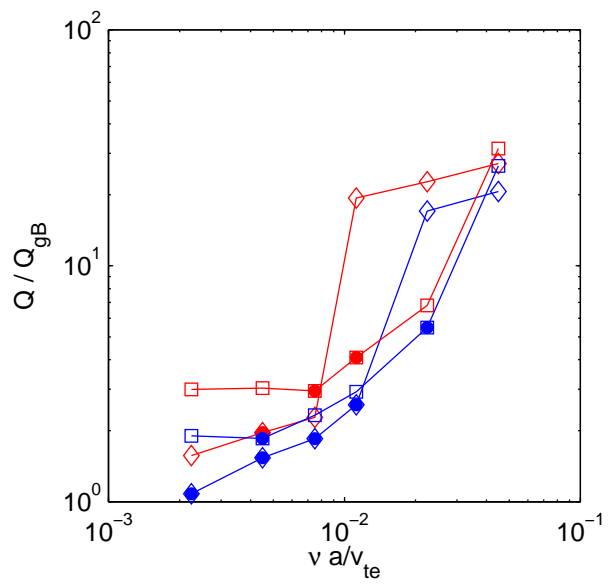

(a)

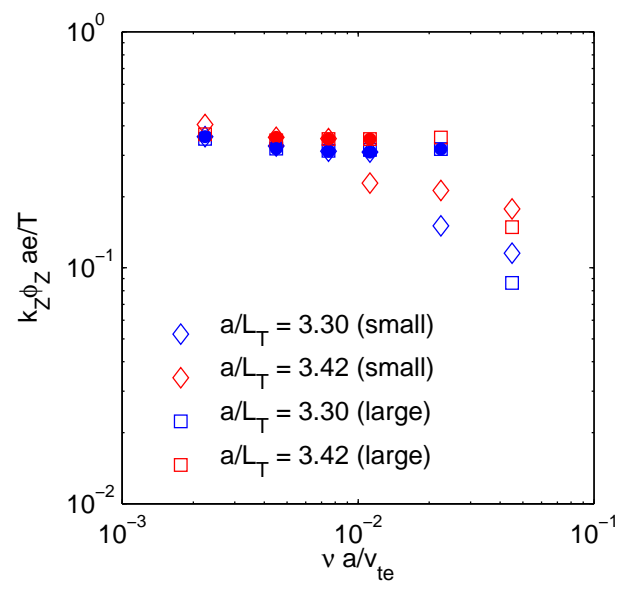

(b)

Figure E1. Variation of (a) the time-averaged normalised electron heat flux $Q / Q_{\mathrm{gB}}$ and (b) the rms zonal velocity $k_{\mathrm{Z}} \phi_{\mathrm{Z}}$, defined by (17), versus normalised electron collisionality $\nu a / v_{t e}$ for our entire simulation series using both small and large boxes, and two temperature gradients, including unresolved runs (hollow symbols; infilled symbols are the resolved points shown in Figure 3).

Note that while these linear simulations were done at zero flow shear, our nonlinear runs had $\gamma_{E}=-0.003 v_{t e} / a$, a value representing the measured rotational shear in the MAST shot on which the rest of our equilibrium parameters were based. The ETG instability in the presence of flow shear can become subcritical in the sense that growth is transient and all modes eventually decay (cf. [64, 65, 66, 67, 68, 69]). Given a finite initial perturbation, such transient growth still leads to a saturated nonlinear state (because long enough transient growth can be as good as a formally unstable mode as far as maintaining turbulence is concerned [65, 66, 67, 69]), although we have found the minimum value of $a / L_{T}$ required for this to be higher than the critical gradient implied by Figure D2: it is typically between 3 and 3.3. The mapping out of a "zero-turbulence manifold" (cf. [70, 71]) for the ETG problem has been left outside the scope of this study — and would be a formidable computational challenge, requiring very many very-long-time simulations. It appears plausible that the key parameters in such a study would be the electron temperature gradient $a / L_{T}$ and collisionality $\nu_{e i} a / v_{t e}$, rather than the ion temperature gradient and the perpendicular flow shear $\gamma_{E}$, which has a more profound effect on the turbulence threshold in the ITG problem $[70,71,69]$. The magnetic geometry factor $q R / r \approx B / B_{p}$, which appears in the scaling (31), may play a similar role — improving confinement as this quantity is reduced in both cases, although for rather different reasons.

\section{Appendix E. Issues of numerical convergence in nonlinear simulations}

We discuss here the selection of runs used to construct Figure 3, and the associated numerical convergence issues. We also include additional evolution plots, complementing the two that were shown in Figure 2. This appendix concludes with a short discussion on the role of flow shear. 


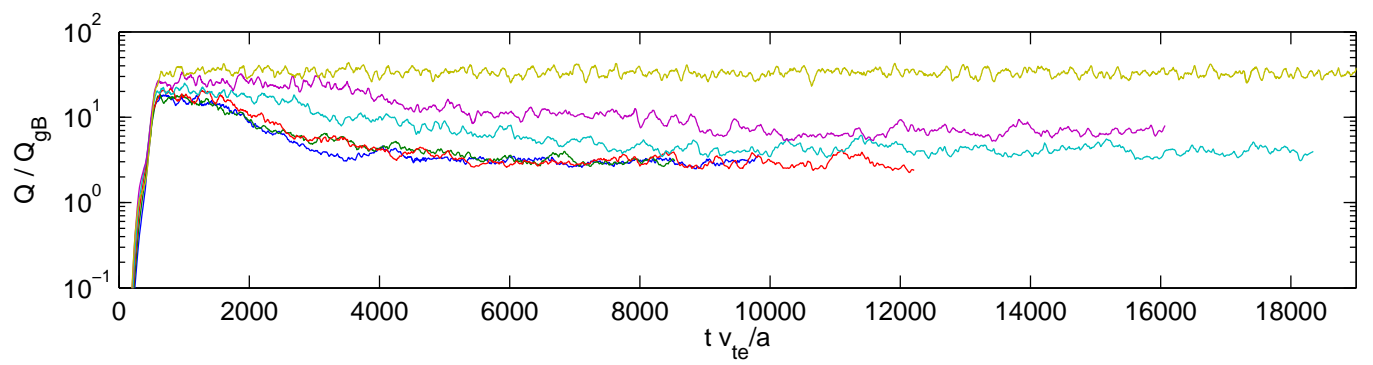

(a)

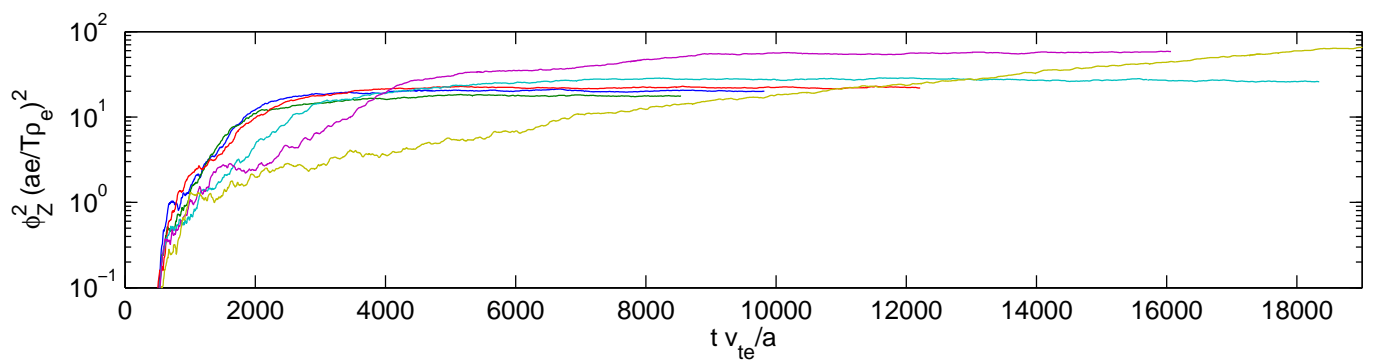

(b)

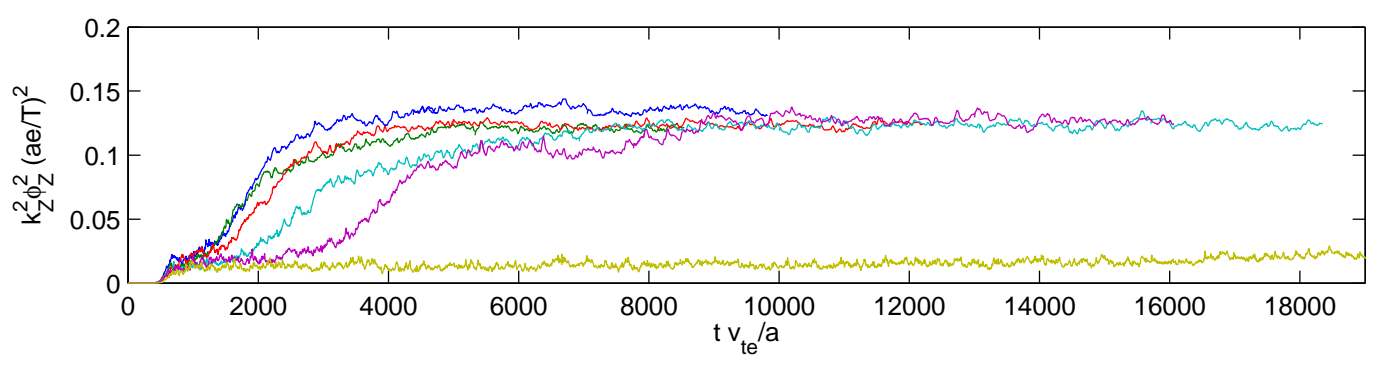

(c)

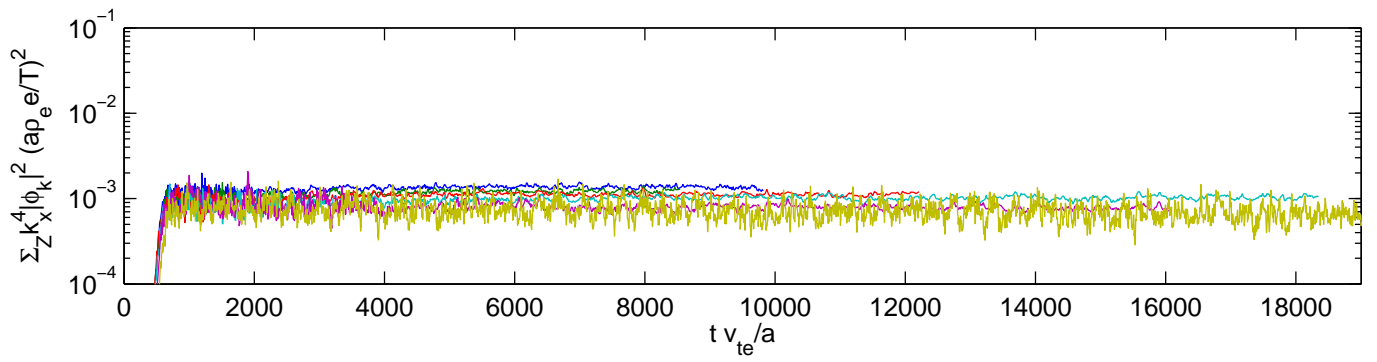

(d)

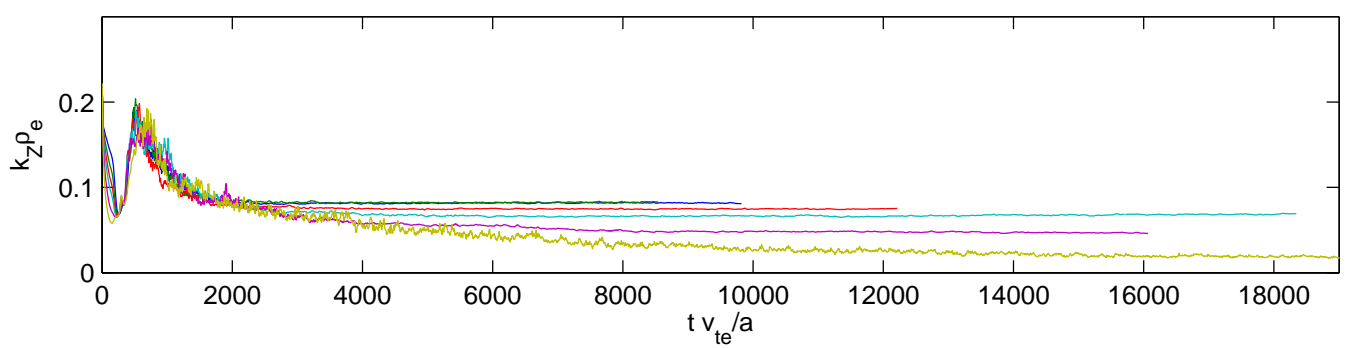

(e)

Figure E2. Evolution in time of (a) the normalised turbulent electron heat flux $Q / Q_{\mathrm{gB}}$, (b) the square of the zonal electrostatic potential, $\phi_{Z}^{2}=\sum_{k_{x}}\left|\phi_{k_{x}, 0}\right|^{2}$, (c) the square of the zonal velocity, $\left(k_{\mathrm{Z}} \phi_{\mathrm{Z}}\right)^{2}=\sum_{k_{x}} k_{x}^{2}\left|\phi_{k_{x}, 0}\right|^{2}$, (d) the square of the zonal shear, $\sum_{k_{x}} k_{x}^{4}\left|\phi_{k_{x}, 0}\right|^{2}$, and (e) the rms zonal wavenumber $k_{\mathrm{Z}}$, for large-box simulations at the nominal $a / L_{T}=3.42$ and at various collisionalities (colours: same as Figure D1, plus yellow for $\nu=2 \nu_{\text {nom }}$ ). 


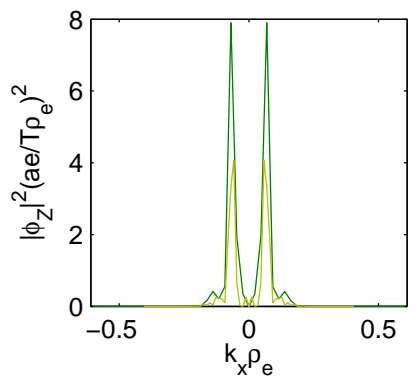

(a)

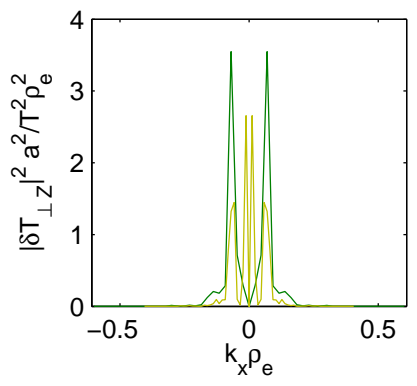

(b)

Figure E3. Spectrum of (a) the zonal potential, (b) the zonal perpendicular temperature perturbation, for $\nu=0.1 \nu_{\text {nom }}, a / L_{T}=3.3$, for a small-box simulation (green) and a largebox simulation (yellow), at the final simulation time in each case.

\section{Appendix E.1. Convergence of the heat flux}

Figure 3 was based on a set of simulations picked from a larger parameter scan that we have carried out. The choice of simulations was based on whether we deemed them to be numerically converged. Here we explain how this was decided.

Figure E1 shows the results of both small- and large-box simulations (see Appendix C.2 for the explanation of what this means), over a range of collisionalities and for two values of the temperature gradient. Runs excluded from Figure 3 are shown as hollow points. They have been excluded because one or more important physical quantity measured in these runs was found to be dominated by either the box scale (the lowest $k$ ) or by grid scales (the highest $k$ 's), and, therefore, the convergence of the simulation with respect to box size and/or spatial resolution could not be relied upon.

Two particular pathologies have been identified, and are also visible in Figure E2, which shows the time evolution of the full set of large-box simulations at the nominal temperature gradient $\left(a / L_{T}=3.42\right)$.

(i) As the collisionality is reduced, it can be seen from the growth of the zonal velocity (Figure E2(c)) that the increasing zonal $\phi^{2}$ (Figure E2(b)) more than compensates initially for the gradually falling zonal wavenumber (Figure E2(e)), until the final level of zonal velocity is reached. However, the zonal shear (Figure E2(d)) remains relatively constant in time. For the highest collisionality (yellow), the heat flux never collapses, and we believe that in this case it is because the required zonal wavenumber is too close to the simulation box scale; see Figure E2(e). Such uncollapsed runs are visible as the high points in Figure E1(a).

(ii) At the low end of the collisionality scale in Figure E2 (blue, red and green), it can be seen that there is no significant difference in the heat fluxes as the collisionality is varied, leading to the plateau of hollow red squares in Figure E1(a) (and a similar plateau for the other temperature gradient, blue squares). These runs are also excluded because of box-scale effects. In these cases, somewhat counterintuitively, the small-box runs, which, however, extend to higher $k$ 's, appear to be resolved and do not exhibit the plateau. Figure E3 shows an example (the lowest-temperaturegradient and lowest-collisionality case), in which the perpendicular temperature is dominated by the box scale for the large-box run, which is excluded.

Eliminating both pathologies gives a fairly clean collisionality scaling when the remaining resolved 


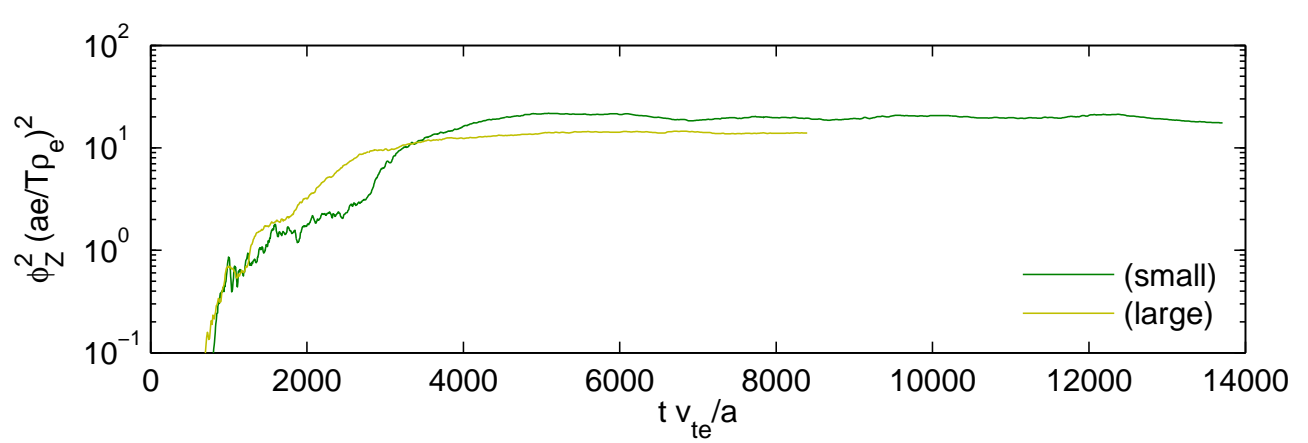

(a)

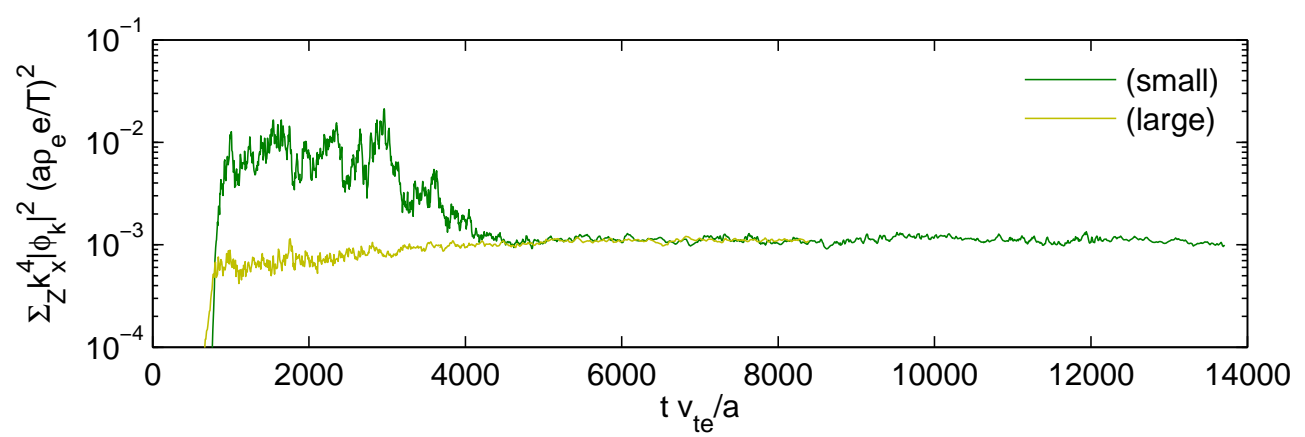

(b)

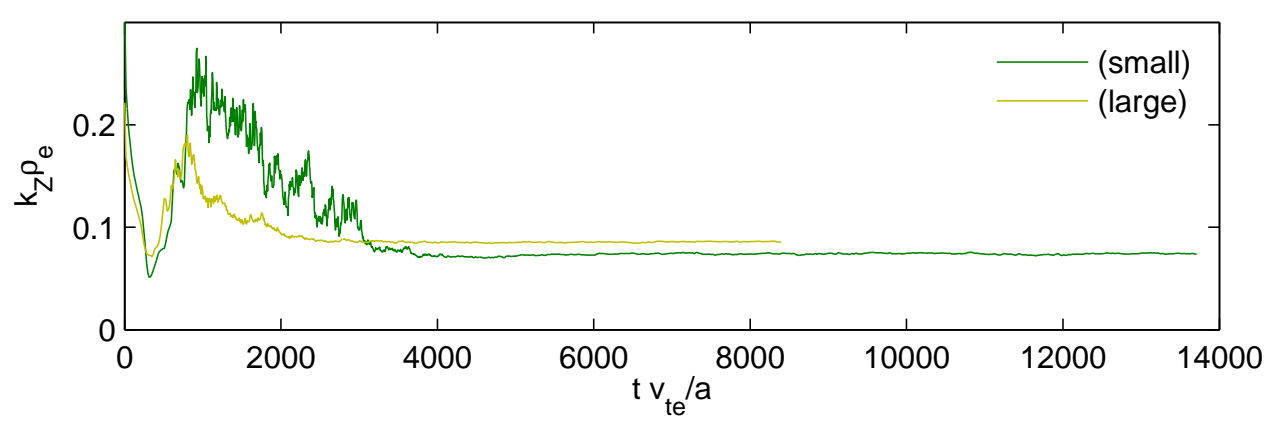

(c)

Figure E4. Evolution in time of (a) the square of the zonal electrostatic potential, $\phi_{Z}^{2}=$ $\sum_{k_{x}}\left|\phi_{k_{x}, 0}\right|^{2}$, (b) the square of the zonal shear, $\sum_{k_{x}} k_{x}^{4}\left|\phi_{k_{x}, 0}\right|^{2}$, and (c) the rms zonal wavenumber $k_{\mathrm{Z}}$, for $\nu=0.2 \nu_{\text {nom }}, a / L_{T}=3.3$ (green: small-box simulations; yellow: large-box simulations). This complements Figure 2.

runs are combined. Given that the zonal damping, according to the scaling demonstrated in section 3.3, is lowest for the box-scale mode, it is not surprising that there are convergence problems at this end of the spectrum. Nonetheless, all the simulations that we consider resolved have saturated with the nonlinear state dominated by zonal modes with wavenumbers above the box-scale mode.

Thus, to summarise, at higher collisionalities, we need larger boxes but can sometimes get away 


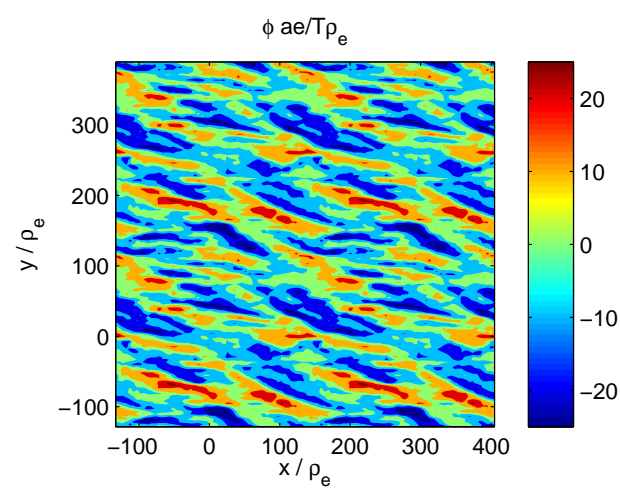

(a)

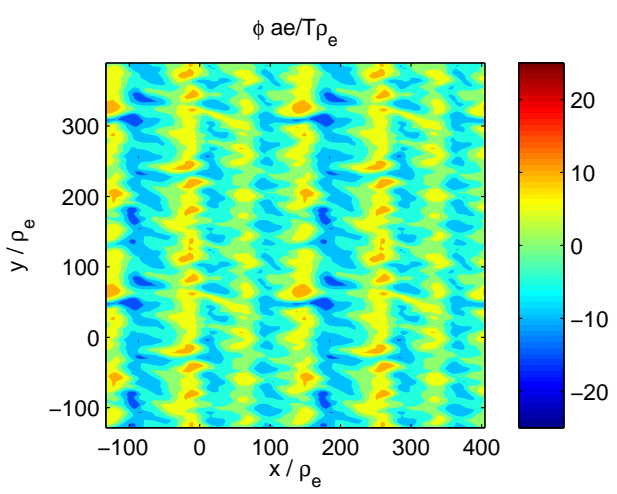

(b)

Figure E5. Electrostatic potential $\phi$ at the outboard midplane, for $\nu=0.2 \nu_{\text {nom }}, a / L_{T}=3.3$ : (a) quasi-saturated state at $t=1204.2 \mathrm{a} / \mathrm{v}_{\text {te }}$, (b) long-time saturated state at $t=7841.5 \mathrm{a} / \mathrm{v}_{\text {te }}$, for small-box simulations showing $2 \times 2$ copies of each periodic domain. The large-box version of this simulation is shown in Figure 1.

with less spatial resolution, whereas at low collisionalities, we need higher spatial resolution, but can sometimes get away with smaller boxes.

For completeness, we include as Figure E4 the time traces of various zonal quantities for the simulations used in Figure 2. When these two figures are combined, the complete set analogous to Figure E2 is obtained. Note that these demonstrate some lack of convergence in the "quasisaturated" state, but the long-time state is resolved. Spatial cross-sections of the electrostatic potential for these two simulations are shown in Figure 1 (large-box) and Figure E5 (small-box). The morphology of the quasi-saturated state is qualitatively distinguishable between the two simulations. This difference is perhaps associated with the clear difference in the zonal shear shown in in Figure E4(b). The small-box simulation has higher spatial resolution, i.e., a higher maximum $k$, and the higher power of $k_{x}$ in the zonal shear (compared to the zonal velocity) emphasises the high end of the spectrum. The long-time saturated states, on the other hand, appear qualitatively the same. Note that in the large-box simulation in Figure 1(b), there are seven periods of the dominant zonal wavelength across the box, whereas in the doubled small box in Figure E5(b) there can only be an even number, which is six (two times the three across each smaller periodic box). This level of discrepancy between the zonal wavenumbers must be expected given the finite domain size.

\section{Appendix E.2. Background flow shear}

Various authors have reported runaway growth in ETG turbulence simulations. To aid saturation, Guttenfelder and Candy [32], following earlier work [72], added background flow shear to their simulations. Roach et al. [28], extending the simulations of Joiner et al. [27], observed runaway growth that was healed by adding either flow shear or sufficient collisions (attributed by them to the stabilisation of weak trapped-electron modes close to the box scale at $k_{y} \rho_{e} \approx 0.02$ ).

In our simulations, we have included an experimentally realistic level of flow shear, $\gamma_{E}=$ $-0.003 v_{t e} / a$, and we do not find runaway behaviour. Note that in all of the cases reported 
above, the normalised perturbed zonal shear (Figure E2(d) and Figure E4(b)) is an order of magnitude larger than the background flow shear. In the "simplified" simulations of section 3.5, zero flow shear was used, and it is reasonable to suppose that the fixed electron-electron collisions helped regularise these simulations over a wider range of collisionalities than was accessible when varying electron-ion and electron-electron collisions together. The simplified simulations support our view that the scaling of the heat flux with the collision rate that we have found does not depend directly on flow shear. Of course, physically, in a real tokamak, flow shear is still needed to suppress the ion-scale turbulence, which we did not model.

\section{Appendix F. Long-time linear damping of the electron zonal modes}

In this Appendix, we derive the scaling (30) of the zonal damping rate.

\section{Appendix F.1. Zonal evolution equation}

Consider the linearised form of the zonal gyrokinetic equation (20) (i.e., ignore its right-hand side). It is convenient to Fourier transform this equation in the field-perpendicular coordinate $x$ (there is no dependence on $y$ by definition of the zonal modes), while leaving the field-parallel derivatives in position space (the zonal modes here are just ones with $k_{y}=0$, but are allowed to have parallel variation). This gives us

$$
\frac{\partial}{\partial t}\left[h+J_{0}\left(k_{\perp} \rho\right) \frac{e \phi}{T} F\right]+v_{\|} \mathbf{b} \cdot \nabla h+i k_{x} v_{B x} h=\left\langle C\left[h e^{-i k_{x} \rho_{x}}\right] e^{i k_{x} \rho_{x}}\right\rangle,
$$

where $J_{0}\left(k_{\perp} \rho\right)$ with $\rho=v_{\perp} / \Omega_{e}$ is the Fourier-space form of the gyroaveraging operator, $k_{\perp}=k_{x}|\nabla x|$ (here the curvilinear coordinate $x$ is defined by (C.2) and so $\nabla x=\left(q / B_{0} r\right) \nabla \psi$ ), $\rho_{x}=(\nabla x) \cdot\left(\mathbf{b} \times \mathbf{v}_{\perp}\right) / \Omega_{e}$, the angle brackets $\langle\ldots\rangle$ denote the averaging of the linearised collision operator over the gyroangle $[73,15]$, and

$$
v_{B x}=\mathbf{v}_{B} \cdot \nabla x=\frac{q}{B_{0} r} \mathbf{v}_{B} \cdot \nabla \psi,
$$

where $\mathbf{v}_{B}$ is given by (7). The quasineutrality equation (14) becomes

$$
\frac{e \phi}{T}\left(1+\frac{1}{\tau}\right)=-\frac{1}{n} \int d^{3} \mathbf{v} J_{0}\left(k_{\perp} \rho\right) h .
$$

\section{Appendix F.2. Long-wavelength ordering and time scales}

Assuming that the zonal modes have long wavelengths, $k_{x} \rho_{e} \ll 1$, we seek the solution to equations (F.1) and (F.3) in the form of an asymptotic expansion

$$
h=h^{(0)}+h^{(1)}+h^{(2)}+\ldots,
$$

where $h^{(n)} \sim\left(k_{x} \rho_{e}\right)^{n} h^{(0)}$. In this ordering, $J_{0}\left(k_{\perp} \rho\right) \approx 1$ to lowest order and clearly, from (F.3), to lowest order, $e \phi / T \sim h^{(0)} / F$. We introduce a formal ordering of time scales in (F.1):

$v_{\|} \mathbf{b} \cdot \nabla \sim \frac{v_{t e}}{a} \sim \nu, \quad k_{x} v_{B x} \sim \frac{k_{x}}{\Omega_{e}} \frac{v_{t e}^{2}}{a}=k_{x} \rho_{e} \frac{v_{t e}}{a} \sim\left(k_{x} \rho_{e}\right) \nu, \quad \frac{\partial}{\partial t} \sim \gamma_{\mathrm{Z}} \sim\left(k_{x} \rho_{e}\right)^{2} \nu$.

This ordering anticipates the kind of solution we are expecting. Generally speaking, any initial zonal perturbation will evolve on three successive time scales: first, it will be damped 
collisionlessly by the streaming and magnetic-drift terms on the time scale $\sim a / v_{t e}$, leaving a finite residual perturbation [24]; then this residual, which is non-Maxwellian, will be damped by collisions on the time scale $\sim 1 / \nu[74,75,76,77]$, leaving a residual perturbed Maxwellian (this will be our $\left.h^{(0)}\right)$; and finally, this perturbed Maxwellian will be damped diffusively at the rate $\gamma_{\mathrm{Z}}$, as we are about to show.

Thus, the zonal perturbation whose evolution we are going to calculate will be close to a steady state, with its evolution essentially governed by the (neoclassical) collisional transport theory.

\section{Appendix F.3. Zeroth order}

With the ordering (F.5), at zeroth order, (F.1) becomes

$$
v_{\|} \mathbf{b} \cdot \nabla h^{(0)}=C\left[h^{(0)}\right] .
$$

If we multiply this equation by $h^{(0)} / F$ and flux-surface average, the left-hand side vanishes (see, e.g., [14], §6.1) and we get

$$
-\overline{\int d^{3} \mathbf{v} \frac{h^{(0)} C_{e e}\left[h^{0}\right]}{F}}+\overline{\int d^{3} \mathbf{v} \frac{\nu_{e i} v_{t e}^{3}}{v^{3} F} \frac{1-\xi^{2}}{2}\left(\frac{\partial h^{(0)}}{\partial \xi}\right)^{2}}=0,
$$

where we have denoted the flux-surface average by an overbar, used the collision operator (15) taken to lowest order in $k_{\perp} \rho_{e}$ and integrated by parts in the term involving electron-ion collisions (cf. [73], §4.2). Both terms in (F.7) are non-negative definite and so must vanish individually. The vanishing of the first of these implies that $h^{(0)}$ must be a perturbed Maxwellian, the vanishing of the second that this perturbed Maxwellian has no mean parallel flow. Therefore,

$$
h^{(0)}=\left[-\frac{e \phi}{T}\left(1+\frac{1}{\tau}\right)+\left(\frac{v^{2}}{v_{t e}^{2}}-\frac{3}{2}\right) \frac{\delta T}{T}\right] F,
$$

where the density perturbation associated with $h^{(0)}$ has been fixed by the requirement that, to lowest order, $h^{(0)}$ and $\phi$ must satisfy (F.3). Note that we omit the superscript (0) on $\phi$ and $\delta T$ because we will not need to calculate these fields explicitly to any higher order.

Finally, substituting (F.8) back into (F.6) and using the fact that, $h^{(0)}$ being a flow-less perturbed Maxwellian, $C\left[h^{(0)}\right]=0$, we find that both $\phi$ and $\delta T$ must be flux functions to zeroth order:

$$
\mathbf{b} \cdot \nabla \frac{e \phi}{T}=\mathbf{b} \cdot \nabla \frac{\delta T}{T}=0
$$

The zeroth-order zonal solution (F.8) is precisely the promised quasi-steady state to which any initial zonal perturbations will relax after initial collisionless and collisional transients.

\section{Appendix F.4. First order}

At first order in $k_{x} \rho_{e}$, equation (F.1) is

$$
v_{\|} \mathbf{b} \cdot \nabla h^{(1)}+i k_{x} v_{B x} h^{(0)}=C\left[h^{(1)}\right] .
$$

Note that any gyroaveraging or FLR effects in the collision operator will only appear in the second order. 
In dealing with (F.10), it is convenient to take advantage of the fact that in tokamaks [78],

$$
\mathbf{v}_{B} \cdot \nabla \psi=-v_{\|} \mathbf{b} \cdot \nabla\left(\frac{v_{\|} I}{\Omega_{e}}\right)
$$

where $I(\psi)=B_{T} R$ is a flux function, $B_{T}$ is the toroidal magnetic field and $R$ the major radius coordinate. Note that $\nabla$ on the right-hand side of (F.11) is taken at constant $\mathcal{E}$ and $\mu$, which, owing to the variation of $B$, is not at constant $v_{\|}$. Using (F.2) and recalling that the safety factor $q(\psi)$ and the radial flux-surface label $r(\psi)$ are both flux functions and the reference magnetic field $B_{0}$ is a constant, we have

$$
v_{B x}=-v_{\|} \mathbf{b} \cdot \nabla\left(\frac{v_{\|}}{\Omega_{e}} \frac{q I}{B_{0} r}\right)=-v_{\|} \mathbf{b} \cdot \nabla\left(\rho_{p e} \frac{v_{\|}}{v_{t e}}\right),
$$

where $\rho_{p e}=\left(q I / B_{0} r\right) \rho_{e}$ is, by definition, the "poloidal gyroradius", so called because $q I / B_{0} r \sim$ $B / B_{p}$, where $B_{p}$ is the poloidal magnetic field. Note that $\Omega_{e}$ and, therefore, $\rho_{e}=v_{t e} / \Omega_{e}$, is calculated using the total, space-dependent field $B$, and so $\rho_{p e}$ is not a flux function.

Since $h^{(0)}$ is a flux function $\left(\mathbf{b} \cdot \nabla h^{(0)}=0\right)$, we may then rewrite (F.10) as follows

$$
v_{\|} \mathbf{b} \cdot \nabla\left(h^{(1)}-i k_{x} \rho_{p e} \frac{v_{\|}}{v_{t e}} h^{(0)}\right)=C\left[h^{(1)}\right] .
$$

The solution of this equation is a standard problem in neoclassical theory [78, 25], but we shall not require its explicit form in order to work out the scalings that we seek. Note from (F.13) that the first-order zonal perturbation has a parallel electron flow (and, therefore, current) of order

$$
\frac{u_{\|}}{v_{t e}} \sim k_{x} \rho_{p e} \frac{\delta p}{p}
$$

where $\delta p / p$ is the zonal pressure perturbation associated with $h^{(0)}$. The perpendicular $\mathbf{E} \times \mathbf{B}$ zonal flow is, of course, just

$$
\frac{v_{E y}}{v_{t e}} \sim k_{x} \rho_{e} \frac{e \phi}{T} .
$$

It is the resistive damping of zonal flows (currents) that will lead to the damping of the zonal modes.

\section{Appendix F.5. Second order}

In order to calculate this damping, we need evolution equations for the zeroth-order fields $\phi$ and $\delta T$. The time derivatives enter at the second order in (F.1). Using (F.8) and (F.12), we have at this order:

$$
\begin{aligned}
& \frac{\partial}{\partial t}\left[-\frac{1}{\tau} \frac{e \phi}{T}+\left(\frac{v^{2}}{v_{t e}^{2}}-\frac{3}{2}\right) \frac{\delta T}{T}\right] F+v_{\|} \mathbf{b} \cdot \nabla h^{(2)}-h^{(1)} v_{\|} \mathbf{b} \cdot \nabla\left(i k_{x} \rho_{p e} \frac{v_{\|}}{v_{t e}}\right) \\
& =k_{x}^{2}\left\langle C\left[h^{(0)} \rho_{x}\right] \rho_{x}\right\rangle+\left\langle C\left[h^{(2)}-\frac{1}{2} k_{x}^{2} \rho_{x}^{2} h^{(0)}\right]\right\rangle
\end{aligned}
$$

where the leading-order FLR parts of the collision operator acting on $h^{(0)}$ have appeared. We now transit-average this equation, integrate the magnetic-drift term by parts and use (F.13) to express $v_{\|} \mathbf{b} \cdot \nabla h^{(1)}$. The result, with the transit-average denoted by overbar, is

$\frac{\partial}{\partial t}\left[-\frac{1}{\tau} \frac{e \phi}{T}+\left(\frac{v^{2}}{v_{t e}^{2}}-\frac{3}{2}\right) \frac{\delta T}{T}\right] F=\overline{-i k_{x} \rho_{p e} \frac{v_{\|}}{v_{t e}} C\left[h^{(1)}\right]+k_{x}^{2}\left\langle C\left[h^{(0)} \rho_{x}\right] \rho_{x}\right\rangle+\langle C[\ldots]\rangle .}$ 
To separate the evolution of $\phi$ and $\delta T$, we take the density and energy moments of (F.17). Integrating it over velocities, and noting that, by conservation of particles, $\int d^{3} \mathbf{v}\langle C[\ldots]\rangle=0$, we get 9

$$
-\frac{n}{\tau} \frac{\partial}{\partial t} \frac{e \phi}{T}=-\overline{-i k_{x} \rho_{p e} \int d^{3} \mathbf{v} \frac{v_{\|}}{v_{t e}} C_{e i}\left[h^{(1)}\right]+k_{x}^{2} \rho_{e}^{2} \int d^{3} \mathbf{v} \frac{C_{e i}\left[h^{(0)} \rho_{x}\right] \rho_{x}}{\rho_{e}^{2}}} .
$$

Only the electron-ion collision terms have survived because, to lowest order, conservation of momentum by the electron-electron collisions implies

$$
\int d^{3} \mathbf{v} v_{\|} C_{e e}\left[h^{(1)}\right]=0, \quad \int d^{3} \mathbf{v} C_{e e}\left[h^{(0)} \rho_{x}\right] \rho_{x}=0 .
$$

In the same vein, multiplying (F.17) by $v^{2} / v_{t e}^{2}$ and integrating over velocities, we find, using energy conservation by collisions, $\int d^{3} \mathbf{v} v^{2}\langle C[\ldots]\rangle=0$,

$$
\frac{3 n}{2} \frac{\partial}{\partial t} \frac{\delta T}{T}=\overline{-i k_{x} \rho_{p e} \int d^{3} \mathbf{v} \frac{v_{\|}}{v_{t e}}\left(\frac{v^{2}}{v_{t e}^{2}}-\frac{3}{2}\right) C\left[h^{(1)}\right]+k_{x}^{2} \rho_{e}^{2} \int d^{3} \mathbf{v} \frac{C\left[h^{(0)} \rho_{x}\right] \rho_{x}}{\rho_{e}^{2}}\left(\frac{v^{2}}{v_{t e}^{2}}-\frac{3}{2}\right)}
$$

The difference between this equation and (F.18) is that the contributions from electron-electron collisions do not vanish (because same-species collisions can support non-zero heat fluxes).

\section{Appendix F.6. Damping rate}

We do not need to solve the neoclassical equation (F.13) for $h^{(1)}$ explicitly to see that, this equation being linear, $h^{(1)}$ will be a linear combination of $e \phi / T$ and $\delta T / T$ with velocity-dependent coefficients, all of which are proportional to $k_{x} \rho_{p e}$; any homogeneous part of $h^{(1)}$ satisfies (F.6) and so can be absorbed into $h^{(0)}$. Therefore, we may schematically represent (F.18) and (F.20) as the following matrix equation at this order: $\dagger$

$$
\begin{aligned}
& \frac{\partial}{\partial t} \frac{e \phi}{T}=\nu_{e i} k_{x}^{2} \rho_{p e}^{2}\left(a_{11} \frac{e \phi}{T}+a_{12} \frac{\delta T}{T}\right) \\
& \frac{\partial}{\partial t} \frac{\delta T}{T}=\nu_{e i} k_{x}^{2} \rho_{p e}^{2}\left(a_{21} \frac{e \phi}{T}+a_{22} \frac{\delta T}{T}\right)+\nu_{e e} k_{x}^{2} \rho_{p e}^{2}\left(b_{21} \frac{e \phi}{T}+b_{22} \frac{\delta T}{T}\right)
\end{aligned}
$$

where $a_{i j}$ and $b_{i j}$ are dimensionless coefficients that depend only on equilibrium parameters. Clearly, if $\nu_{e i}=0$, the matrix is defective (has a zero row corresponding to the upper equation (F.21)) and the damping rate of both the potential and temperature perturbations of the slowest damped eigenmode vanishes. Therefore,

$$
\gamma_{\mathrm{Z}} \propto \nu_{e i} k_{x}^{2} \rho_{p e}^{2}
$$

The order-unity numerical prefator in the exact expression for $\gamma_{Z}$ depends on the ratio $\nu_{e e} / \nu_{e i}$, which itself is order unity. Thus, we have shown that the long-time damping rate of the zonal modes always satisfies (30), a scaling that is indeed well reproduced in our numerical simulations (Figure 4).

व The last term in (F.18) is related to the spatial FLR diffusion term that we wrote out explicitly in the gyroaveraged collision operator (15).

$\dagger$ Note that in the absence of magnetic drifts, in a straight field, $h^{(1)}=0$ and we must replace $\rho_{p e}$ with $\rho_{e}$ everywhere, with the collisional evolution of the zonal modes now controlled by the FLR spatial-diffusion terms in the gyrokinetic collision operator. 
Appendix F.7. Zonal damping by same-species collisions

The result (30) survives even if, artifically, the ratio $\nu_{e e} / \nu_{e i}$ is made large - as was done in the "simplified" simulations of section 3.5. In this case, (F.22) simply implies that

$$
\frac{\delta T}{T} \approx-\frac{b_{21}}{b_{22}} \frac{e \phi}{T}
$$

and, according to (F.21), the zonal perturbation is still damped at the rate $\gamma_{\mathrm{Z}} \sim \nu_{e i} k_{x}^{2} \rho_{p e}^{2}$.

If we consider an even more artificial situation in which $\nu_{e i}$ is made to vanish completely, we must go to next order when calculating the density moment (F.18) of the zonal kinetic equation (F.1) to allow higher-order finite-drift-orbit-width FLR terms to come in. These terms will be small by an extra factor of $\left(k_{x} \rho_{p e}\right)^{2}$ and so technically we would have to reorder the time derivative two powers of $k_{x} \rho_{e}$ higher and then work through two extra orders in our expansion. $\ddagger$ It is clear though that the result of this amounts to replacing (F.21) and (F.22) with

$$
\begin{aligned}
& \frac{\partial}{\partial t} \frac{e \phi}{T}=\nu_{e e} k_{x}^{4} \rho_{p e}^{4}\left(b_{11} \frac{e \phi}{T}+b_{12} \frac{\delta T}{T}\right), \\
& \frac{\partial}{\partial t} \frac{\delta T}{T}=\nu_{e e} k_{x}^{2} \rho_{p e}^{2}\left(b_{21} \frac{e \phi}{T}+b_{22} \frac{\delta T}{T}\right),
\end{aligned}
$$

using (F.26) to infer again the coupling (F.24) between the zonal potential and temperature perturbations, and finally arriving at a zonal damping rate

$$
\gamma_{\mathrm{Z}} \sim \nu_{e e} k_{x}^{4} \rho_{p e}^{4}
$$

This is the scaling that is indeed obtained numerically when $\nu_{e i}$ is switched off (black crosses in Figure 4).

These considerations might appear moot, as $\nu_{e i}=0$ is unphysical and achievable only in numerical experiments. They do, however, help us gain some insight into the way in which our theoretical aruments would be modified if we applied them to zonal dynamics in ITG rather than ETG turbulence, as suggested in section 4.2.

\section{Appendix F.8. Case of ion zonal modes}

The only differences between the linearised gyrokinetic equation for the ion zonal modes and (F.1) are a minus sign in front of the $e \phi / T$ term, accounting for the ion charge, and the absence of interspecies collisions:

$$
\frac{\partial}{\partial t}\left[h_{i}-J_{0}\left(k_{\perp} \rho\right) \frac{e \phi}{T_{i}} F\right]+v_{\|} \mathbf{b} \cdot \nabla h_{i}+i k_{x} v_{B x} h_{i}=\left\langle C_{i i}\left[h_{i} e^{-i k_{x} \rho_{x}}\right] e^{i k_{x} \rho_{x}}\right\rangle .
$$

Another very important difference between the ion and electron cases is the Boltzmannelectron closure (32), which removes the flux-surface-averaged part of the zonal potential from the electron-density response. Combining (32) with the expression (12) for the ion density perturbation in terms of $h_{i}$, we get the following version of the quasineutrality equation:

$$
\frac{e \phi}{T_{i}}+\frac{e(\phi-\bar{\phi})}{T_{e}}=\frac{1}{n} \int d^{3} \mathbf{v} J_{0}\left(k_{\perp} \rho\right) h_{i} .
$$

That the difference between (F.29) and (F.3) is significant becomes obvious if we take the density moment of (F.28), i.e., integrate it over velocities keeping $\mathbf{r}$ constant (equivalently, multiply by

$\ddagger$ This was indeed confirmed independently by such a calculation [79]. 
$J_{0}\left(k_{\perp} \rho\right)$ and integrate over $\left.\mathbf{v}\right)$, and then flux-surface average. To lowest order in $k_{\perp} \rho_{i}$, the term in the square brackets becomes, using (F.29),

$$
\overline{\frac{1}{n} \int d^{3} \mathbf{v} J_{0}\left(k_{\perp} \rho\right)\left[h_{i}-J_{0}\left(k_{\perp} \rho\right) \frac{e \phi}{T_{i}} F\right]} \approx \frac{1}{2} k_{\perp}^{2} \rho_{i}^{2} \frac{e \bar{\phi}}{T_{i}} .
$$

This has two extra powers of $k_{x}$ compared to the analogous term for electron zonal flows (see the left-hand side of (F.18)).

By a calculation analogous to Appendix F.3, the zeroth-order solution $h_{i}^{(0)}$ of (F.28) in the longtime limit must again be a quasi-steady (i.e., slow-evolving), constant on flux surfaces, flow-less perturbed Maxwellian. The rest of the calculation proceeds similarly to the electron case with the exception that only momentum-conserving ion-ion collisions are present and so the velocity integral of the collision terms in the density equation will be smaller by an extra factor of $k_{x}^{2} \rho_{p i}^{2}$, as explained in Appendix F.7. In view of (F.30), the analog of (F.25) for ion zonal modes will then be

$$
\frac{\partial}{\partial t} \frac{1}{2} k_{x}^{2} \rho_{p i}^{2} \frac{e \phi}{T_{i}}=\nu_{i i} k_{x}^{4} \rho_{p i}^{4}\left(c_{11} \frac{e \phi}{T_{i}}+c_{12} \frac{\delta T_{i}}{T_{i}}\right),
$$

where we have absorbed all factors accounting for differences between $k_{\perp}$ and $k_{x}$ and between $\rho_{i}$ and $\rho_{p i}$ into the dimensionless coefficients $c_{11}$ and $c_{12}$. The extra factors of $k_{x}^{2} \rho_{p i}^{2}$ on the left-hand side (due to the special role of flux-surface-averaged potential in the Boltzmann-electron closure) and on the right-hand side (due to momentum conservation by the ion-ion collision operator) cancel, leaving $e \phi / T_{i}$ to evolve on the diffusive time scale $\sim 1 / \nu_{i i} k_{x}^{2} \rho_{p i}^{2}$. The evolution equation for $\delta T_{i} / T_{i}$ does not have these extra factors on either side and so feature the same time scale. Thus, the ion zonal modes, similarly to the electron ones (although for a different reason), will be damped at a rate

$$
\gamma_{\mathrm{Z} i} \sim \nu_{i i} k_{x}^{2} \rho_{p i}^{2}
$$

We remind the reader that the above calculation, like the whole of Appendix F, is concerned solely with the linear physics of the zonal modes considered on their own. Its significance nonlinearly will depend upon the interactions between these modes and the nonzonal modes. In the main part of the paper, we report nonlinear results for ETG turbulence only, but we do discuss ITG turbulence further, in this wider nonlinear context, in the concluding section 4.2 .

\section{References}

[1] Valovič M, Akers R, de Bock M, McCone J, Garzotti L, Michael C, Naylor G, Patel A, Roach C, Scannell R, Turnyanskiy M, Wisse M, Guttenfelder W, Candy J and the MAST Team 2011 Nuclear Fusion $\mathbf{5 1}$ 073045

[2] Kotschenreuther M, Rewoldt G and Tang W 1995 Computer Physics Communications 88128

[3] GS2 is an open-source Fortran code URL http://gyrokinetics.sourceforge.net/

[4] Doerk H, Jenko F, Pueschel M J and Hatch D R 2011 Physical Review Letters 106155003

[5] Guttenfelder W, Candy J, Kaye S M, Nevins W M, Wang E, Bell R E, Hammett G W, Leblanc B P, Mikkelsen D R and Yuh H 2011 Physical Review Letters 106155004

[6] Field A R, Akers R J, Brickley C, Carolan P G, Challis C, Conway N J, Cunningham G, Meyer H, Roach C M, Walsh M J and the MAST Team 2004 Core Heat Transport in the MAST Spherical Tokamak Proceedings of 20th IAEA Fusion Energy Conference, Vilamoura, Portugal, EX/P2-11

[7] Roach C M, Applegate D J, Connor J W, Cowley S C, Dorland W D, Hastie R J, Joiner N, Saarelma S, Schekochihin A A, Akers R J, Brickley C, Field A R, Valovic M and the MAST Team 2005 Plasma Physics and Controlled Fusion 47 B323 
[8] Field A R, Michael C, Akers R J, Candy J, Colyer G, Guttenfelder W, Kim Y C, Roach C M, Saarelma S and the MAST Team 2010 Plasma rotation and transport in the MAST spherical tokamak Proceedings of 23rd IAEA Fusion Energy Conference, Daejon, Republic of Korea, EXC/P8-04

[9] Field A, Michael C, Akers R, Candy J, Colyer G, Guttenfelder W, c Ghim Y, Roach C, Saarelma S and the MAST Team 2011 Nuclear Fusion 51063006

[10] Dorland W, Jenko F, Kotschenreuther M and Rogers B N 2000 Physical Review Letters 855579

[11] Jenko F, Dorland W, Kotschenreuther M and Rogers B N 2000 Physics of Plasmas 71904

[12] Roach C M, Walters M, Budny R V, Imbeaux F, Fredian T W, Greenwald M, Stillerman J A, Alexander D A, Carlsson J, Cary J R, Ryter F, Stober J, Gohil P, Greenfield C, Murakami M, Bracco G, Esposito B, Romanelli M, Parail V, Stubberfield P, Voitsekhovitch I, Brickley C, Field A R, Sakamoto Y, Fujita T, Fukuda T, Hayashi N, Hogeweij G M D, Chudnovskiy A, Kinerva N A, Kessel C E, Aniel T, Hoang G T, Ongena J, Doyle E J, Houlberg W A, Polevoi A R, ITPA Confinement Database and Modelling Topical Group and ITPA Transport Physics Topical Group 2008 Nuclear Fusion 48125001

[13] Frieman E A and Chen L 1982 Physics of Fluids 25502

[14] Abel I G, Plunk G G, Wang E, Barnes M, Cowley S C, Dorland W and Schekochihin A A 2013 Reports on Progress in Physics $\mathbf{7 6} 116201$

[15] Abel I G, Barnes M, Cowley S C, Dorland W and Schekochihin A A 2008 Physics of Plasmas 15122509

[16] Barnes M, Abel I G, Dorland W, Ernst D R, Hammett G W, Ricci P, Rogers B N, Schekochihin A A and Tatsuno T 2009 Physics of Plasmas 16072107

[17] Barnes M, Abel I G, Dorland W, Görler T, Hammett G W and Jenko F 2010 Physics of Plasmas 17056109

[18] Sugama H, Watanabe T H and Nunami M 2009 Physics of Plasmas 16112503

[19] Schekochihin A A, Parker J T, Highcock E G, Dellar P J, Dorland W and Hammett G W 2016 Journal of Plasma Physics 82905820212

[20] Barnes M, Parra F I and Schekochihin A A 2011 Physical Review Letters 107115003

[21] Ghim Y c, Schekochihin A A, Field A R, Abel I G, Barnes M, Colyer G, Cowley S C, Parra F I, Dunai D and Zoletnik S 2013 Physical Review Letters 110145002

[22] Wesson J 2004 Tokamaks (Oxford: Oxford University Press) chap 4.18, p 209 3rd ed

[23] Rogers B N, Dorland W and Kotschenreuther M 2000 Physical Review Letters 855336

[24] Rosenbluth M N and Hinton F L 1998 Physical Review Letters 80724

[25] Helander P and Sigmar D J 2005 Collisional Transport in Magnetized Plasmas (Cambridge: Cambridge University Press)

[26] Diamond P H, Itoh S I, Itoh K and Hahm T S 2005 Plasma Physics and Controlled Fusion 4735

[27] Joiner N, Applegate D, Cowley S C, Dorland W and Roach C M 2006 Plasma Physics and Controlled Fusion 48685

[28] Roach C M, Abel I G, Akers R J, Arter W, Barnes M, Camenen Y, Casson F J, Colyer G, Connor J W, Cowley S C, Dickinson D, Dorland W, Field A R, Guttenfelder W, Hammett G W, Hastie R J, Highcock E, Loureiro N F, Peeters A G, Reshko M, Saarelma S, Schekochihin A A, Valovic M and Wilson H R 2009 Plasma Physics and Controlled Fusion $\mathbf{5 1} 124020$

[29] Parker J B and Krommes J A 2013 Physics of Plasmas 20100703

[30] Parker J B and Krommes J A 2014 New Journal of Physics 16035006

[31] Mantica P, Angioni C, Baiocchi B, Baruzzo M, Beurskens M N A, Bizarro J P S, Budny R V, Buratti P, Casati A, Challis C, Citrin J, Colyer G, Crisanti F, Figueiredo A C A, Frassinetti L, Giroud C, Hawkes N, Hobirk J, Joffrin E, Johnson T, Lerche E, Migliano P, Naulin V, Peeters A G, Rewoldt G, Ryter F, Salmi A, Sartori R, Sozzi C, Staebler G, Strintzi D, Tala T, Tsalas M, Eester D V, Versloot T, deVries P C, Weiland J and JET EFDA Contributors 2011 Plasma Physics and Controlled Fusion 53124033

[32] Guttenfelder W and Candy J 2011 Physics of Plasmas 18022506

[33] Nakata M, Watanabe T H, Sugama H and Horton W 2010 Physics of Plasmas 17042306

[34] Idomura Y, Tokuda S and Kishimoto Y 2005 Nuclear Fusion 451571

[35] Idomura Y 2006 Physics of Plasmas 13080701

[36] Hillesheim J C, Dickinson D, Roach C M, Saarelma S, Scannell R, Kirk A, Crocker N A, Peebles W A, Meyer H and the MAST Team 2016 Plasma Physics and Controlled Fusion 58014020

[37] Gurchenko A D and Gusakov E Z 2010 Plasma Physics and Controlled Fusion 52124035

[38] Guttenfelder W, Candy J, Kaye S M, Nevins W M, Bell R E, Hammett G W, LeBlanc B P and Yuh H 2012 Physics of Plasmas 19022506

[39] Guttenfelder W, Peterson J, Candy J, Kaye S, Ren Y, Bell R, Hammett G, LeBlanc B, Mikkelsen D, Nevins W and Yuh H 2013 Nuclear Fusion 53093022

[40] Kaye S M, Levinton F M, Stutman D, Tritz K, Yuh H, Bell M G, Bell R E, Domier C W, Gates D, Horton W, Kim J, LeBlanc B P, Luhmann Jr N C, Maingi R, Mazzucato E, Menard J E, Mikkelsen D, Mueller D, Park H, Rewoldt G, Sabbagh S A, Smith D R and Wang W 2007 Nuclear Fusion 47499

[41] ITER Physics Expert Groups on Confinement and Transport and Confinement Modelling and Database, and ITER Physics Basis Editors 1999 Nuclear Fusion 392175

[42] Cordey J, Thomsen K, Chudnovskiy A, Kardaun O, Takizuka T, Snipes J, Greenwald M, Sugiyama L, Ryter 
F, Kus A, Stober J, DeBoo J, Petty C, Bracco G, Romanelli M, Cui Z, Liu Y, McDonald D, Meakins A, Miura Y, Shinohara K, Tsuzuki K, Kamada Y, Urano H, Valovic M, Akers R, Brickley C, Sykes A, Walsh M, Kaye S, Bush C, Hogewei D, Martin Y, Cote A, Pacher G, Ongena J, Imbeaux F, Hoang G, Lebedev S and Leonov V 2005 Nuclear Fusion 451078

[43] Bourdelle C, Gerbaud T, Vermare L, Casati A, Aniel T, Artaud J, Basiuk V, Bucalossi J, Clairet F, Corre Y, Devynck P, Falchetto G, Fenzi C, Garbet X, Guirlet R, Gürcan Ö, Heuraux S, Hennequin P, Hoang G, Imbeaux F, Manenc L, Monier-Garbet P, Moreau P, Sabot R, Ségui J L, Sirinelli A, Villegas D and the Tore Supra Team 2011 Nuclear Fusion 51063037

[44] Dorland W and Hammett G W 1993 Physics of Fluids B 5812

[45] Hammett G W, Beer M A, Dorland W, Cowley S C and Smith S A 1993 Plasma Physics and Controlled Fusion 35973

[46] Abel I G and Cowley S C 2013 New Journal of Physics 15023041

[47] Cowley S C, Kulsrud R M and Sudan R 1991 Physics of Fluids B 32767

[48] Nevins W M, Candy J, Cowley S, Dannert T, Dimits A, Dorland W, Estrada-Mila C, Hammett G W, Jenko F, Pueschel M J and Shumaker D E 2006 Physics of Plasmas 13122306

[49] Jenko F and Dorland W 2002 Physical Review Letters 89225001

[50] Dimits A M, Bateman G, Beer M A, Cohen B I, Dorland W, Hammett G W, Kim C, Kinsey J E, Kotschenreuther M, Kritz A H, Lao L L, Mandrekas J, Nevins W M, Parker S E, Redd A J, Shumaker D E, Sydora R and Weiland J 2000 Physics of Plasmas 7969

[51] Ricci P, Rogers B N and Dorland W 2006 Physical Review Letters 97245001

[52] Lin Z, Hahm T S, Lee W W, Tang W M and Diamond P H 1999 Physical Review Letters 833645

[53] Lin Z, Hahm T S, Lee W W, Tang W M and White R B 2000 Physics of Plasmas 71857

[54] Mikkelsen D R and Dorland W 2008 Physical Review Letters 101135003

[55] Miller R L, Chu M S, Greene J M, Lin-Liu Y R and Waltz R E 1998 Physics of Plasmas 5973

[56] McCone J 2011 Impurity Density and Poloidal Rotation Measurements on MAST Ph.D. thesis National University of Ireland

[57] Garzotti L, Figueiredo J, Roach C M, Valovič M, Dickinson D, Naylor G, Romanelli M, Scannell R, Szepesi G and the MAST Team 2014 Plasma Physics and Controlled Fusion 56035004

[58] Parra F I, Barnes M and Peeters A G 2011 Physics of Plasmas 18062501

[59] Beer M A, Cowley S C and Hammett G W 1995 Physics of Plasmas 22687

[60] Highcock E 2012 The Zero-Turbulence Manifold in Fusion Plasmas Ph.D. thesis University of Oxford (arXiv:1207.4419)

[61] Beer M A 1995 Gyrofluid Models of Turbulent Transport in Tokamaks Ph.D. thesis Princeton University

[62] Hammett $\mathrm{G}$ and Roach $\mathrm{C}$ private communication

[63] Jenko F, Dorland W and Hammett G W 2001 Physics of Plasmas 84096

[64] Newton S L, Cowley S C and Loureiro N F 2010 Plasma Physics and Controlled Fusion 52125001

[65] Highcock E G, Barnes M, Schekochihin A A, Parra F I, Roach C M and Cowley S C 2010 Physical Review Letters 105215003

[66] Barnes M, Parra F I, Highcock E G, Schekochihin A A, Cowley S C and Roach C M 2011 Physical Review Letters 106175004

[67] Highcock E G, Barnes M, Parra F I, Schekochihin A A, Roach C M and Cowley S C 2011 Physics of Plasmas 18102304

[68] Schekochihin A A, Highcock E G and Cowley S C 2012 Plasma Physics and Controlled Fusion 54055011

[69] van Wyk F, Highcock E G, Schekochihin A A, Roach C M, Field A R and Parra F I 2016 In preparation

[70] Highcock E G, Schekochihin A A, Cowley S C, Barnes M, Parra F I, Roach C M and Dorland W 2012 Physical Review Letters 109265001

[71] Ghim Y c, Field A R, Schekochihin A A, Highcock E G, Michael C and the MAST Team 2014 Nuclear Fusion 54042003

[72] Candy J, Waltz R E, Fahey M R and Holland C 2007 Plasma Physics and Controlled Fusion 491209

[73] Schekochihin A A, Cowley S C, Dorland W, Hammett G W, Howes G G, Quataert E and Tatsuno T 2009 Astrophysical Journal Supplement Series 182310

[74] Hinton F L and Rosenbluth M N 1999 Plasma Physics and Controlled Fusion 41 A653

[75] Kim E J, Holland C and Diamond P H 2003 Physical Review Letters 91075003

[76] Xiao Y, Catto P J and Molvig K 2007 Physics of Plasmas 14032302

[77] Xiao Y, Catto P J and Dorland W 2007 Physics of Plasmas 14055910

[78] Hinton F L and Wong S K 1985 Physics of Fluids 283082

[79] Connor J private communication 